\title{
Composite Portfolio Performance: An Investigation into Indian Mutual Funds
}

\author{
P. Janaki Ramudu* and Krishna Kumar
}

Alliance University School of Business, Bangalore, India

\begin{abstract}
Earlier in the 1960s, though they were aware of the concept of risk, the portfolio managers did not know as to how to measure and hence their performance was measured only in terms of rate of return. Though quite a few measures were developed in 1960s, it was Friend, Blume and Crockett who developed a mechanism to group portfolios into similar risk class. This in fact helped the portfolio managers to compare the performance of various funds more meaningfully in terms of risk-return relationship. Keeping the importance of two sides of investment coin: the Risk and the Return, we, in this paper attempted to analyze the performance of equity linked and diversified funds. We also tested if the portfolio managers' stock selection ability enhanced the performance. We have used measures like Treynor's, Sharpe's, Jensen's Alpha, the Information Ratio and Net Selectivity. Using these measures, we attempted to find out if the portfolio managers could generate aboveaverage rate of return for a given risk class. The sample comprised equity linked savings and diversified funds in Indian context. The analysis was done on quarterly, half yearly, yearly and five yearly basis for each fund. This facilitated us to identify if the time factor played a role in the performance of a given fund. The results revealed that the performance of the fund managers primarily depended on the type of measure. While the fund(s) performed better according to a given method, than that of others in a given risk class, it was vice versa according to other measures. This reveals that the selection of performance measure matters a lot while assessing the performance of a fund. Analysis of Variance (ANOVA) revealed that the performance of a fund depended on time factor also. The results of our study carry very significant implications with respect to portfolio performance analysis.
\end{abstract}

Keywords: Portfolio Performance, Net Selectivity, Portfolio Beta, Portfolio Standard Deviation, Portfolio Alpha

\section{Introduction and Review of Literature}

'Unity Creates Strength' is the quote that stands as the foundation for the emergence of mutual funds. While there is no proof with $100 \%$ reliability as to when and how the mutual funds industry emerged, it is believed that King William I launched first mutual fund in the Netherlands in 1822. A few others also believe that Adriaan Van Ketwich, a Dutch merchant, created the investment trust and gave an idea to King William I to establish the concept of diversification. It is also believed that this idea of diversification would have come up primarily to increase the appeal of investments to smaller investors with highly limited capital. The idea of pooling the resources and spreading the risk component came up in Britain and France which in turn moved on to United States. The first closed-end fund was formed by Boston Personal Property Trust way back in 1893. However, the creation of the Alexander Fund in Philadelphia in 1893 remains as an important milestone in the history of mutual funds. Followed by this, creation of the Massachusetts Investors' Trust in Boston in 1924, which eventually went public in 1928 laid the foundation for wide spread popularity of modern

*Email: janakiramudu.p@alliance.edu.in 
mutual funds industry. With not many developments after this, the period between 1960 and 1969 saw the launching of many mutual fund schemes by several companies which resulted in huge inflow and out flow of funds through mutual funds vehicle. A few firms like, Wells Fargo thereafter laid the strongest foundation for index fund. With bull market mania in US markets, in 1980s the fund managers like Max Heine, Michael Price and Peter Lynch became very popular as top fund managers. Though the mutual funds industry took off in a stunning way, it was not free of scandals. The burst of technology bubbles made the investment in mutual funds as not always benign but could malign at times. Though there were mutual funds scandals and global financial crisis in 2003 and 2008-2009 respectively, the industry is still growing at a reasonable rate.

\subsection{Mutual Funds in India}

As a result of the far sighted vision of the then finance minister, T T Krishnamachari, and the prime minister Jawaharlal Nehru, Unit Trust of India was formed with an enactment of UTI Act 1963 in the parliament. Though Reserve Bank of India had the control over it in the initial stage, Industrial Development Bank of India (IDBI) took over the control in 1978. Entry of public sector funds in 1987 raised the quantum of assets under management of Rs. 6,700 crores in 1988 by UTI to the tune of Rs. 47,004 crores by the end of the year 1993. These figures translate into Compounded Annual Growth Rate (CAGR) of $47.64 \%$. This indicates a phenomenal growth rate in mutual funds industry in India. A few of the popular public sector undertakings that contributed to such a rate are Life Insurance Corporation of India (LIC), State Bank of India (SBI) and General Insurance Corporation of India (GIC). In order to boost the industry further, private sector funds and foreign mutual funds were allowed to enter the industry since 1993 which resulted in further enhancement of assets under management to the extent of Rs. 1,21,805 crores held by around thirty three mutual funds by the end of the year 2003. However, the CAGR in assets under management between 1993 and 2003 was only $10 \%$ being lower than that of between the years 1988 and 1993. During these phases it was only UTI which had a lion share in assets under management (Rs. 44,541 crore by the end of the year 2003). As it was reported by Centre for Monitoring Indian Economy (CMIE), total assets under management in India touched all time high of Rs. 8.68 trillion by the end of May 2013. This implies a CAGR of $21.7 \%$ in assets under management between the years 1993 and 2013 which is almost double the rate that of between the years 1988 and 1993. The observed trend in the growth of mutual funds industry in India reveals its importance and role that it plays in Indian investment world. This also implies that the investors on an aggregate basis want to spread the risk across many less risky assets than that of only equity.

The increasing magnitude of focus on mutual funds, as discussed in earlier paragraphs, implies a lot to the fund managers as well as the investors in terms of risk-return relationship. As it is known unlike equity, investment in mutual funds is less absorbent to the sudden market shocks. The prime reason adduced to this is diversification of funds across various asset classes. The risk and the return are the two sides of an investment coin. While assessing the performance of a mutual fund manager, the risk return relationship is a key aspect and hence the returns of a portfolio should always be adjusted to the risk. In the absence of risk measurement tools, the return alone was used to assess the performance. This may make sense if the investor does not bother about the volatility of the returns. But in the light of enhanced awareness and competition, volatility in the returns gained prominence over a period of time and today the risk is in-separable from the return. However there is no any common agreement among the experts about the way the volatility or so called risk is measured in an investment. While a few believed in standard deviation (or variance which measures total risk) others believed only in nondiversifiable risk which is a part of total risk. While there are various schools of thought as to how to measure a fund's performance, the most popular ones that are in vogue are Sharpe ratio, Treynor ratio, Jensen's alpha, Modigliani squared $\left(\mathrm{M}^{2}\right)$ and Information ratio (also known as appraisal ratio). The use of any specific measure would depend on examination of circumstances. We will elaborate on this aspect in a later section called research methodology. 
The paper is organized into four sections, dealing with introduction and literature review (section I), objectives, methodology and hypotheses (section II), results, discussions and findings (section III) and conclusions and scope for further study (section IV).

Having understood the importance of investment in mutual funds and the measurement of fund's performance, we turn our focus onto the research available in this area. For a better understanding of the developments, we have reviewed the research papers in chronological order. We also tried our level best to focus more on the developments in portfolio performance during the recent past. In fact, the concept of risk-adjusted performance evaluation came into limelight along with popularly known Capital Asset Pricing Model (CAPM) way back in 1966.

Treynor (1965) and Sharpe (1966) used mean variance to measure the performance, recognizing the implications of CAPM, Michael Jensen (1969) used excess of actual return of the portfolio over its CAPM based expected one. He coined this excess return as "Alpha" of the portfolio. Followed by these developments, the academicians started investigating, at an increasing rate, into the performance of various mutual funds.

Examining the relationship between market timing skills and the performance of mutual fund, Chang and Lewellen (1984) concluded that attribution of the performance is always a subjective matter. They evidenced that the collective performance of the fund is relevant to efficient market hypothesis. This in turn reveals that the implications of efficient hypothesis and differential investment strategies of the manager matter a lot while assessing and attributing the performance.

Testing the Efficient Market Theory (EMT), Ippolito (1993) evidenced in his study that incurring more expenses on research and trading in strong form of the market is a matter of wasting resources as it never resulted in beating the market. His findings were in line with the hypothesis that the fund managers are successful in finding new information and using such information for generating return to offset fund expenses. He attributed his findings to modified version of EMT proposed by Grossman and Stiglitz (1980).

Investigating about the mutual fund managers' attitude towards risk and the return, Vos (1997) found that $40 \%$ of the sample investors did not believe the rates of return published by the most of the funds. They identified that the fund managers did little to incorporate the risk into the return while reporting their performance. This finding reveals that the performance as reported by the fund manager may not fully be reliable and hence there always exists the need for the investors to re-look into such reported risk-adjusted performance measures.

Measuring mutual fund performance with characteristic-based benchmarks, Daniel et al. (1997) evaluated the performance of 125 passive funds and evidenced selectivity ability of fund managers but no timing ability. The findings of their study, like that of many, reveal that the fund managers could not possess any market timing skills in order to optimize the portfolio return.

Studying the effect of stock selection, trading, cost reduction, marketing and risk taking ability on the performance of the fund, Ang et al. (1998) evidenced that in case of poorly performing funds high frequency trading and more risk of loss lead towards lower returns while expense reduction ability lead to higher portfolio returns. The study revealed very weak relationship between selectivity and performance.

Focusing on style investing of 770 actively managed funds, Indro Daniel et al. (1998) evidenced that the funds that changed the style strategies performed worst when compared to the ones that were stable in style. They also found that large-cap funds were the most mean-variance efficient style consistent when compared the S\&P 500 index.

Testing the market timing abilities of 570 funds during 1987-1996, Rao (2000) found that majority of the fund managers did not possess market timing skills even in 
bull market. The results of this study did not support the hypothesis that the fund managers have the ability to successfully time their portfolio returns on the market portfolio return.

Yet in another study with respect to the link between style and performance, Davis (2001) also evidenced that the long-term performance of the fund was not persistent with style investing. He however found some evidence that style had some impact on shortterm performance.

Taking top five growth funds and ten index funds in India, Patil and Rao (2011) analyzed the performance during the years 2007 through 2010. They found out that the mutual funds performed better while compared to other investments like equity and bonds portfolios.

Examining if Indian fund managers could select and time the market correctly, Chopra (2011) measured the performance of 36 mutual funds in India during the years 2001 through 2009. Their study revealed little evidence that any specific fund manager could use timing skills to outperform the market.

Huang, Sialm and Zhang (2011) attempted to investigate into the effect of risk shifting behavior of fund managers on the performance. Having used holdingsbased measures as the representation of risk shifting, they found that the funds that increased the risk did perform worse than the funds that kept the risk level stable over a period of time. Their study thus suggest that risk shifting behavior is an indication of inferior ability or may be, the fund managers could get motivated to do so by agency costs. This paper holds greater significance in terms of studying the risk return relationship of funds.

Cuthbertson, Nitzsche and O'Sullivan (2012) in their study on UK mutual funds evidenced that most of the funds could not perform better than the market and instead there was false discovery rate in the performance of the funds. Using false discovery rate as the measure they evidenced that only $3.7 \%$ of the total funds in UK outperformed the market.
Based on the monthly data of twenty eight equity diversified Indian funds, Kumar (2012) evidenced that 60\% of the select fund managers were not able to time and beat the market only in terms of security selection. The study revealed that almost all the fund managers failed to exhibit the skills to time the market and enhance the portfolio return.

Barron and Ni (2013) investigated the effect of Morningstar's rating on the replacement of fund manager. They found that mutual fund investors respond positively to the Morningstar measure of performance and therefore the Morningstar rating was considered as the better predictor of fund manager replacement.

\section{Objectives, Methodology and Hypotheses of the Study}

Considering the importance of portfolio performance evaluation as evidenced in earlier section, the study aims:

1. To Investigate and empirically analyze quarterly, half yearly, yearly, three yearly and five yearly performance analysis of select mutual funds in India and trace out if the frequency matters in performance measurement.

2. To compare traditional versus composite performance measurements and trace out the contrast and implications.

3. To empirically prove if there were any specific funds the performance of which was superior according to all or at least major measures.

4. To investigate if the performance of sample funds was consistent over the years 2008-09 through 2012-12.

The measures used to measure and analyze the performance of mutual funds are as follows.

\begin{tabular}{cl}
\hline \multicolumn{1}{c}{ Measure } & \multicolumn{1}{c}{ Equation } \\
\hline Sharpe Ratio (S.R) & $\left(\mathrm{R}_{\mathrm{p}}-\mathrm{R}_{\mathrm{f}}\right) / \sigma_{\mathrm{p}}$ \\
& $(\mathrm{EQ} .1)$ \\
& $\left(\mathrm{R}_{\mathrm{p}}-\mathrm{R}_{\mathrm{f}}\right) / \beta_{\mathrm{p}}$ \\
Treynor Ratio (T.R) & $(\mathrm{EQ} .2)$
\end{tabular}




\begin{tabular}{|c|c|}
\hline Jensen's Alpha (J.A) & $\begin{array}{l}\alpha_{p}=R_{p}-\left\{R_{f}+\beta_{p}\right. \\
\left.\left(R_{m}-R_{f}\right)\right\} \\
(E Q .3)\end{array}$ \\
\hline Information Ratio (I.R) & $\begin{array}{l}\alpha_{\mathrm{p}} / \sigma\left(\mathrm{e}_{\mathrm{p}}\right) \\
\text { (EQ. 4) }\end{array}$ \\
\hline Modigliani Squared $\left(\mathrm{M}^{2}\right)$ & $\begin{array}{l}\mathrm{R}_{\mathrm{p}^{*}}-\mathrm{R}_{\mathrm{m}} \\
\text { (EQ. 5) }\end{array}$ \\
\hline $\begin{array}{l}\text { Fama's Net Selectivity } \\
\text { (N.S) }\end{array}$ & $\begin{array}{l}\mathrm{R}_{\mathrm{p}}-\mathrm{R}_{\mathrm{m}}\left(\sigma\left(\mathrm{R}_{\mathrm{p}}\right)\right) \\
\text { (EQ. 7) }\end{array}$ \\
\hline
\end{tabular}

where,

$\mathrm{R}_{\mathrm{p}}=$ Portfolio average return

$\mathrm{R}_{\mathrm{f}}=\quad$ Average risk free return

$\sigma_{\mathrm{p}}=$ portfolio standard deviation

$\beta_{\mathrm{p}}=$ portfolio beta

$\alpha_{\mathrm{p}}=$ portfolio alpha (portfolio excess return over expected return)

$\mathrm{R}_{\mathrm{p}^{*}}=$ adjusted portfolio return

$\sigma\left(e_{p}\right)=$ portfolio un-systematic or tracking error

Though we presume that every finance professional would be aware of the above measures and terms involved in, we would like to throw spme light on the differences and implications of the above measures for better understanding. While Sharpe and Treynor measure the performance almost on similar grounds they vary in terms of risk adjustment. Sharpe takes total risk $(\sigma)$ into account and Treynor considers only systematic risk ( $\beta$ ). Picking up on Sharpe's CAPM based expected return; Jensen looks into the excess of actual return over such expected return. Jensen's alpha thus indicates and helps to find out market imperfections if any in the market. While higher the alpha better it is for the investor, such higher and lower alphas reveal the stocks/portfolios being undervalued and overvalued. Jensen's alpha is also used to test if CAPM holds good to any specific security.Again taking on Sharpe's ratio, Goodwin(1998) noted that Sharpe ratio is a special case of Information Ratio (IR) or also known as Appraisal Ratio (AR). The information ratio divides portfolio excess return by non-systematic risk $\left(\sigma\left(\mathrm{e}_{\mathrm{p}}\right)\right.$ also known as tracking error. The credit of information ratio development however goes to Treynor and Black (1973). Another measure as equivalent to Sharpe's measure developed by
Graham and Harvey (1994) is $\mathrm{M}^{2}$ (for Modigliani squared). They suggested looking into diversifiable portion of the risk in a portfolio by comparing the standard deviation of portfolio return with that of market. If there is any possibility of diversifying portfolio non-systematic risk, we need to adjust the portfolio accordingly and there by compare the adjusted portfolio return with of market. If adjusted portfolio return is more than market return, it is understood as the portfolio did perform better than the market and vice-versa. Despite having these many measures, the performance of the fund may also depend on many other factors, circumstances in which a fund is operated and frequency of the time during which performance is assessed. It may therefore be noted that there is no single method which is perfect and no performance measurement is hundred percent reliable. These measures only provide a particular direction to understand the performance of a fund in comparison with the market and its peer group funds.

Apart from the above measures and equations, we also use multiple ' $R$ ' in order to study the extent of correlation between the performance of the fund and the market and $\mathrm{R}^{2}$ to find out the explained and unexplained portions of the volatility. Out of many a fund in India, we have taken fifty star rated funds at random for study purpose falling in eight different indices (or Benchmark). Most of the funds selected fall in BSE 100 and BSE 200 indices while there was only one fund each from BSE 500 and BSE Midcap indices. The list of the selected funds and the index concerned could be found in Annexure ' $A$ '. The data required has been sourced from 'navindia.com' for the financial years 2008-09 through 2012-13. We presume that five years data is sufficient enough for the study in order to bring out the latest trends in the performance of the sample funds. We have analyzed the performance of the funds in the frequencies of quarterly, half yearly, yearly and five yearly. We also have computed the returns in terms of two yearly and three yearly and five yearly rolling Compounded Annual Growth Rates (CAGR). However, when it comes to the performance measurement using various ratios (like Sharpe Ratio, Treynor Ratio, and Jensen's Alpha etc.) we have computed on yearly basis. Thus, we have attempted to analyze the performance in 
terms of pure return (without adjusting to the risk) and risk-adjusted return. This facilitates us also to compare and see as to how traditional performance measurement differs from composite performance measurement. We have captured the computations in form of tables and graphs. As a part of the study, we test the following hypotheses at $5 \%$ significance level through one-way ANOVA. MS-Excel software has been used extensively to compute various ratios and test the hypotheses. It may please be noted that instead of pasting entire table, we have taken only observed ' $\mathrm{P}$ ' value form ANOVA table concerned in case of each hypothesis.

$\mathrm{H}_{1}$ : Yearly mean returns (Average Return, A.R) of sample mutual funds in India did not vary over the years 2008-09 and 2012-13 (i.e. $A \cdot R_{50,2008-2009}=A \cdot R_{50 \text {, }}$ $\left.{ }_{2009-2010}=A \cdot R_{50,2010-2011}=A \cdot R_{50,2011-2012}=A \cdot R_{50,2012-2013}\right)$

$\mathrm{HO}_{2}$ : Mean Sharpe Ratio of sample mutual funds in India did not vary over the years 2008-09 and 2012-13 (i.e. $S . R_{50,2008-2009}=S . R_{50,2009-2010}=S . R_{50,2010-2011}=S . R_{50 \text {, }}$ 2011-2012 $=S \cdot R_{50,2012-2013}$ )

$\mathrm{HO}_{3}$ : Mean Treynor Ratio of sample mutual funds in India did not vary over the years 2008-09 through 2012-13 (i.e. $T . R_{50,2008-2009}=T . R_{50,2009-2010}=T . R_{50,2010-}$ $\left.{ }_{2011}=T \cdot R_{50,2011-2012}=T \cdot R_{50,2012-2013}\right)$

$\mathrm{HO}_{4}$ : Mean Jensen's Alpha of sample mutual funds in India did not vary over the years 2008-09 through 2012-13 (i.e. Alpha $a_{50,2008-2009}=$ Alpha $_{50,2009-2010}=$ Alpha $_{50,2010-2011}=$ Alpha $_{50,2011-2012}=$ Alpha $\left._{50,2012-2013}\right)$

$\mathrm{HO}_{5}$ : Mean Information Ratio (I.R) of sample mutual funds in India did not vary over the years 2008-09 through 2012-13 (i.e. $I . R_{50,2008-2009}=I \cdot R_{50,2009-2010}=$ $\left.I \cdot R_{50,2010-2011}=I \cdot R_{50,2011-2012}=I \cdot R_{50,2012-2013}\right)$

$\mathrm{H}_{6}$ : Mean Modigliani Squared $\left(\mathrm{M}^{2}\right)$ of sample mutual funds in India did not vary over the years 2008-09 through 2012-13 (i.e. $M^{2}{ }_{50,2008-2009}=M_{50,2009-2010}^{2}=M^{2}$, 2010-2011 $\left.=M_{50,2011-2012}^{2}=M_{50,2012-2013}^{2}\right)$

$\mathrm{HO}_{7}$ : Mean Net selectivity (N.S) of sample mutual funds in India did not vary over the years 2008-09 through 2012-13 (i.e. N.S $S_{50,2008-2009}=N . S_{50,2009-2010}=$ $\left.N . S_{50,2010-2011}=N . S_{50,2011-2012}=N . S_{50,2012-2013}\right)$.

\section{Results, Discussions and Findings}

As we mentioned earlier, we first discuss the quarterly performance of sample funds over 2008-09 through 2012-13.

\subsection{Quarterly performance}

The quarterly performance computations of sample and benchmark funds are summarized in Table 1. As we observe, all sample funds and benchmark funds have performed exceptionally high during the first two quarters of the financial year 2009-10, while they performed negatively during all the quarters of the fiscal 2008-09. Also third and fourth quarters of the fiscal 2010-11 and first three quarters of the fiscal 2011-12 were found to be very bad for all the funds including benchmark funds. Considering the quarterly average during 2008-09 through 2012-13 a few funds like ICIC Discovery fund, Reliance Equity Opportunity fund, IDFC Premier Equity fund and DSPBR Small \& Midcap fund have performed better than other funds. The quarterly mean values of the returns indicate that all the funds including benchmark funds have generated positive return. There is of course a serious limitation of high volatility of the returns during the quarters. When we look into fifty funds quarterly average returns of sample funds, it was in Q1 and Q2 of the fiscal 2009-10 and Q4 of the fiscal 2011-12 the returns were very high. Barring these quarters, the funds' average return during the rest of the quarters was very bad. The computations pertaining to benchmark funds reveal that all the benchmark funds have performed badly except for the first three quarters of the year 2009-10. Barring these three quarters, the performance in the rest of the quarters is not impressive at all. The quarterly average return of the benchmark funds has also been on the lower side compared to that of sample funds. Table 1 provides very meaningful information to understand as to how each and every fund has performed over all the quarters during the years 2008-09-2012-13. We believe this information is of great use to the concerned in terms of diagnosing the past performance of the sample and benchmark funds on quarterly basis. 


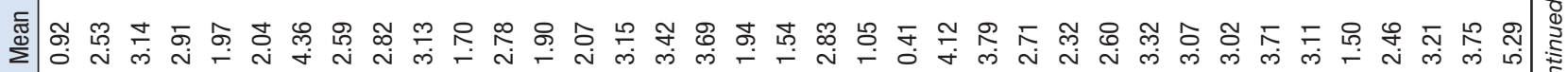

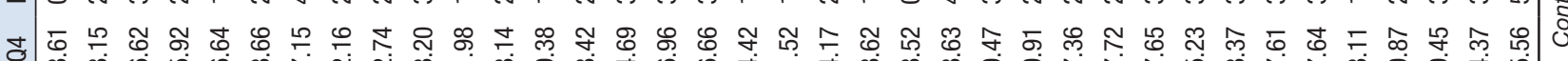

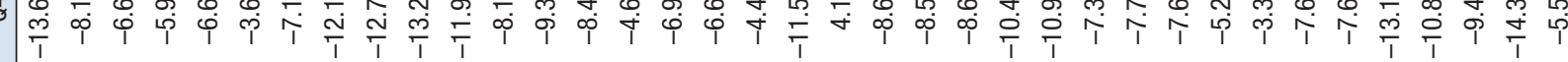

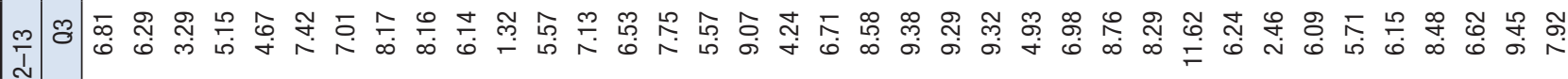
忒 శ m б

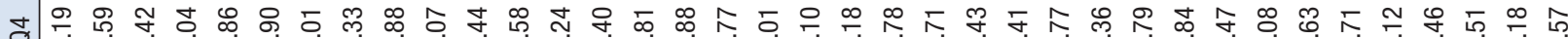

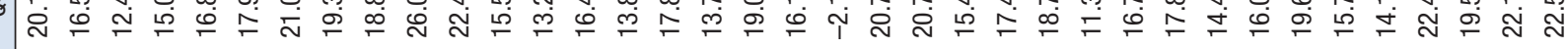
ஜ

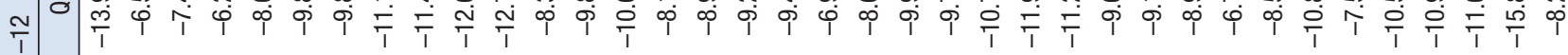

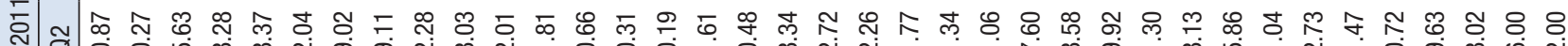
宁宁

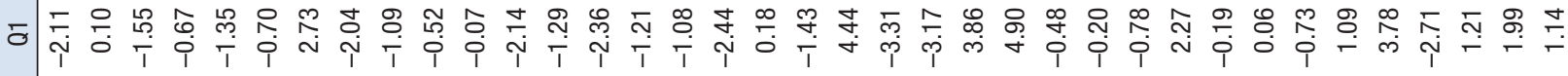

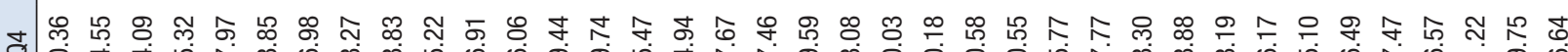

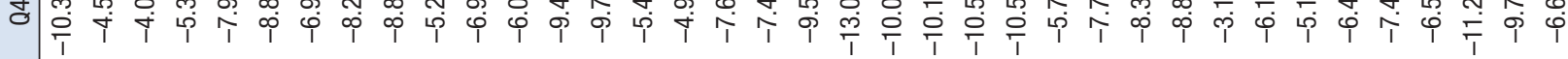
=

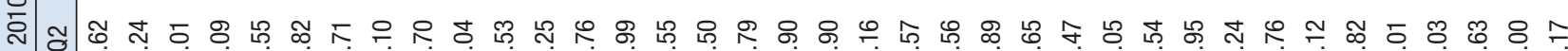

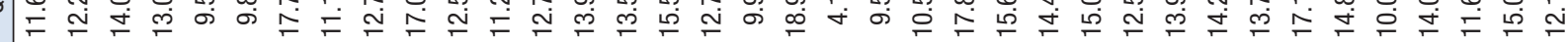

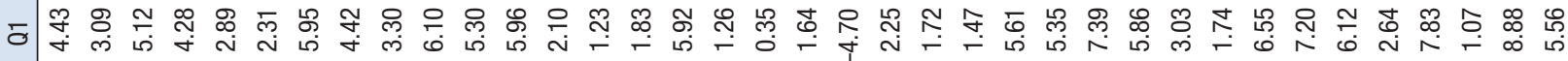

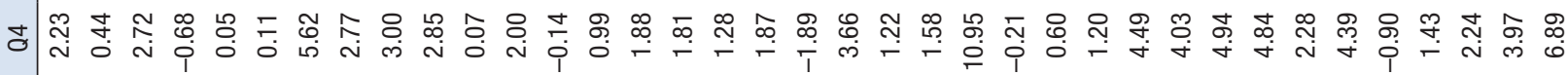
○

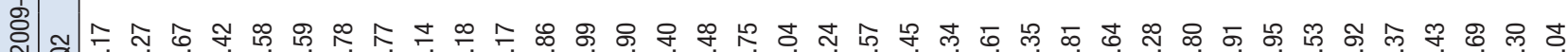

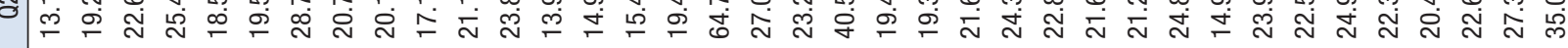

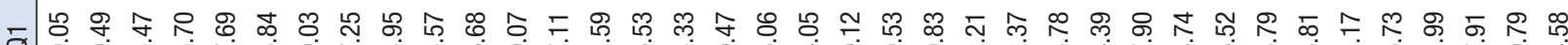

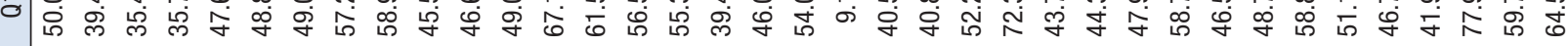
J 屉

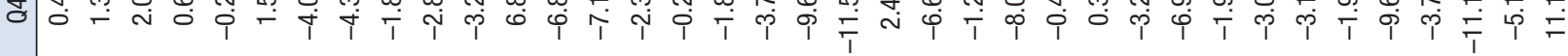
象

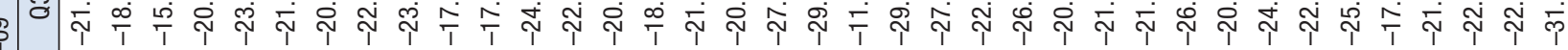

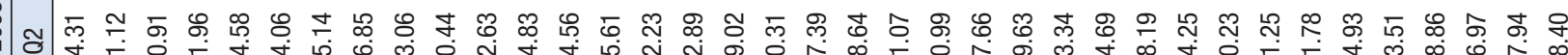

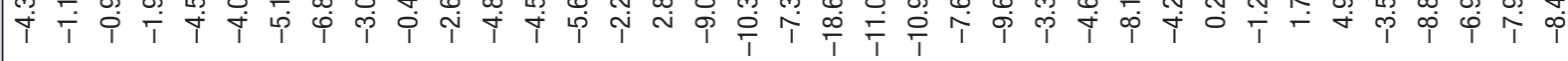

б

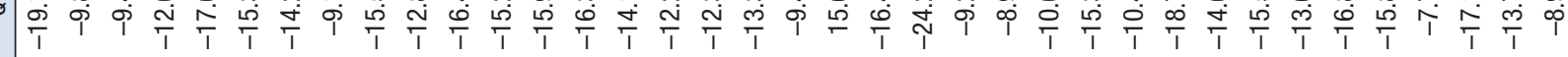




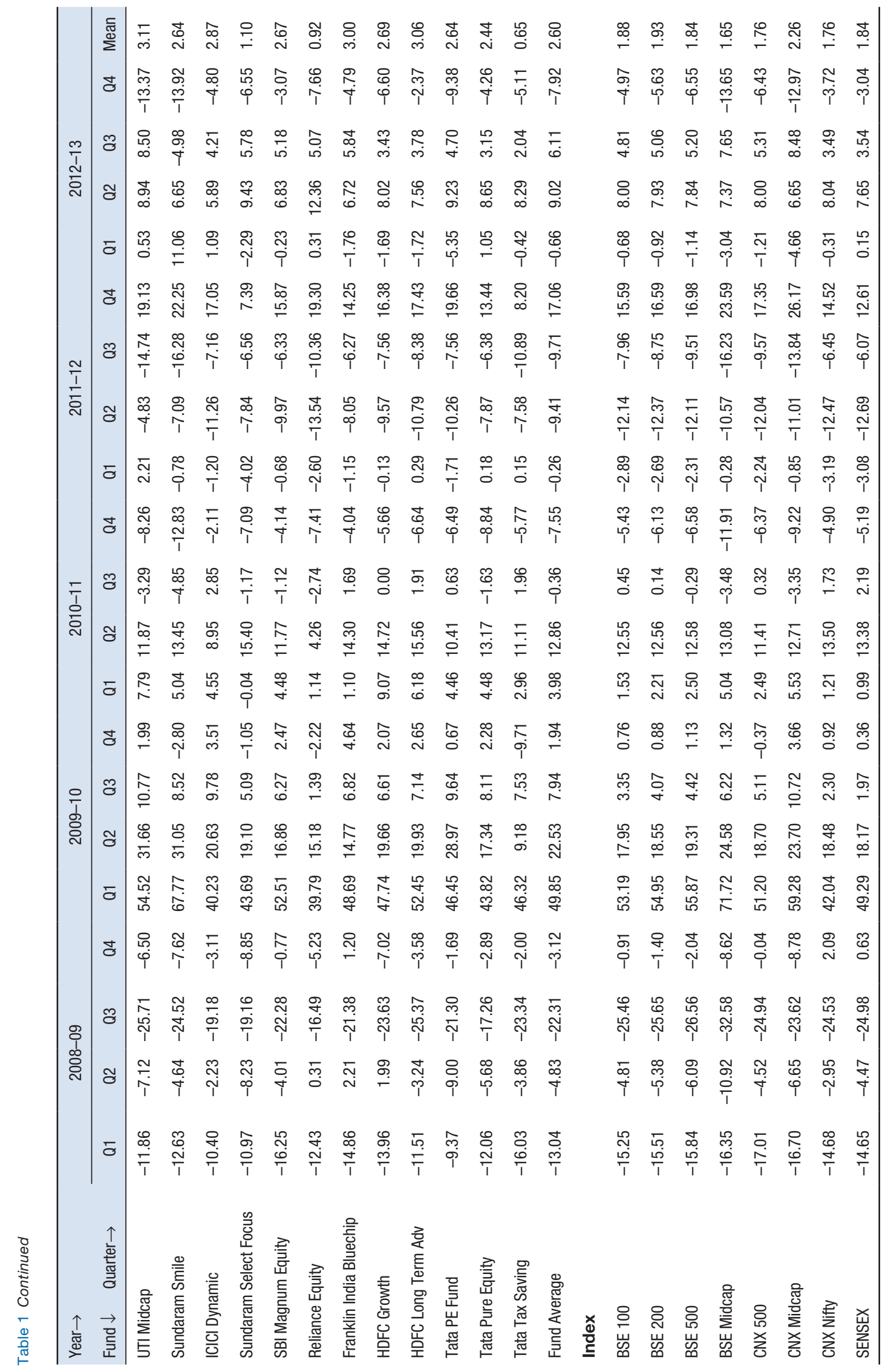




\subsection{Half Yearly Performance}

Table 2 contains the data pertaining half yearly performance of sample and bench mark funds. The data in Table 2 reveals all sample and benchmark funds have performed extremely well during $\mathrm{H} 1, \mathrm{H} 2$ of the fiscal 2009-10, and H1 of the fiscal 2010-11 followed by reasonable performance during $\mathrm{H} 2$ of 2011-12 and $\mathrm{H} 1$ of 2012-13. The performance of all the funds has been found to be very bad with very high negative returns particularly in $\mathrm{H} 1$ and $\mathrm{H} 2$ of the year 2008-09. Like in the case of mean return of all quarters (Table 1), the mean return of the halves of all the years indicate that it were ICICI Discovery fund, Reliance Equity Opportunity fund, IDFC Classic Equity fund and DSPBR Small \& Midcap fund that topped the list. The key finding here is thus these funds have performed consistently on both quarterly and half-yearly basis. The data in Table 2 also reveal that all the funds, including benchmark ones, have performed very well on an aggregate basis. Again, like in case of quarterly performance, this is primarily due to exceptionally high returns in the year 2009-10. When we focus on the half yearly performance of benchmark funds (Table 2), we notice that all the funds have performed equally well on an aggregate basis. This better performance again is due to exceptionally high returns in 2009-10. We also observe that there is no fund, either sample or benchmark, that yielded positive return during $\mathrm{H} 1$ and $\mathrm{H} 2$ of 2008-09. Also same is the case during $\mathrm{H} 2$ of 2010-11 and $\mathrm{H} 1$ of 2011-12 barring ICICI Dynamic fund. The key observation out of half yearly performance analysis is the performance of a few funds has been the same when compared to that of quarterly. However bench mark funds performed better on half yearly basis when compared to that of quarterly. Table 2 thus provides summary of half yearly performance of sample and benchmark funds which, we feel, may be useful in diagnosing the performance of the funds on half yearly basis and then compare with quarterly performance in Table 1 .

\subsection{Yearly Performance}

Having made some key observations out of quarterly and half yearly performance of the funds, we now turn our focus on to yearly performance measurement and analysis. The relevant data in this regard is summarized in Table 3. Like in the case of quarterly and half yearly performance, all the funds have done extremely bad in the year 2008-09 and extremely well in the year 2009-10. When we observe the data in Table 3 the return was as low as $-56.01 \%$ in 2008-09 (in case of ICICI Midcap fund) and as high as $159.35 \%$ in 2009-10 (in case of ICICI Discovery fund). It may be noted that these two funds fall under same Asset Management Company (AMC). This kind of extremes could be found even in case of many funds. Similar to quarterly and half yearly returns, ICICI Discovery fund, Reliance Equity Opportunity fund and IDFC Premier fund have topped the list in terms of yearly performance. This reveals that these funds have exhibited consistent performance irrespective of measurement frequency. A few funds that fall in such category are Sundaram Select Midcap, Franklin India Prima, UTI Dividend Yield, SBI Magnum Equity, Birla Advantage, ICICI Midcap and Sundaram Growth Fund. In case of other funds there was however some deviation in the quarterly, half yearly and yearly performance. We therefore observe that measurement frequency may not matter in case of a few funds and it matters in case of majority of the funds. As we mentioned earlier we tested if the yearly mean return of sample funds varied across study period. As calculated ' $p$ ' value $(0.00)$ is less than the critical 'p' (0.05), we reject $\mathrm{H}_{1}$ and conclude that the mean return of sample funds varied over the years 2008-09 and 2012-13. This implies that the time factor played a role in the returns generated by the by the sample funds over a period of time.

\subsection{Rolling Compounded Annual Growth Rate}

Apart from quarterly, half yearly and yearly analysis, we also have computed rolling compounded annual growth rates in the returns of both sample funds and benchmark funds. The superiority of rolling returns over yearly return is that the rolling return reflects the cumulative return on a continuously investment over a period of time. We have computed the CAGR in rolling returns on two yearly $(2008-10,2009-11,2010-12$ and 2011-13), three yearly (2008-11, 2009-12 and 2010-13) and five yearly (2008-2013) bases. This sort of computation is useful to the investors to know as to what could have been the return if the investment was held for a specified period. The results are summarized 
Table 2. Half yearly returns (in \%) of sample and index funds

\begin{tabular}{|c|c|c|c|c|c|c|c|c|c|c|c|}
\hline \multirow{2}{*}{$\begin{array}{l}\text { Year } \rightarrow \\
\text { Fund } \downarrow\end{array}$} & \multicolumn{2}{|c|}{ 2008-09 } & \multicolumn{2}{|c|}{ 2009-10 } & \multicolumn{2}{|c|}{ 2010-11 } & \multicolumn{2}{|c|}{ 2011-12 } & \multicolumn{2}{|c|}{ 2012-13 } & \multirow[b]{2}{*}{ Mean } \\
\hline & $\mathrm{H} 1$ & $\mathrm{H} 2$ & $\mathrm{H} 1$ & H2 & $\mathrm{H} 1$ & H2 & $\mathrm{H} 1$ & $\mathrm{H} 2$ & $\mathrm{H} 1$ & $\mathrm{H} 2$ & \\
\hline DSPBR Tiger & -22.63 & -21.21 & 69.81 & 5.43 & 16.57 & -14.41 & -12.75 & 3.49 & 6.41 & -7.73 & 2.30 \\
\hline DSPBR Top 100 & -10.85 & -17.46 & 66.37 & 5.55 & 15.71 & -4.08 & -10.18 & 9.00 & 5.13 & -2.37 & 5.68 \\
\hline UTI Dividend Yield & -10.31 & -13.44 & 66.18 & 12.56 & 19.84 & -3.18 & -7.09 & 4.03 & 5.38 & -3.55 & 7.04 \\
\hline UTI Equity & -13.76 & -19.69 & 70.19 & 7.33 & 17.93 & -2.60 & -8.89 & 7.91 & 9.25 & -1.08 & 6.66 \\
\hline SBI Magnum Tax Gain & -20.85 & -23.53 & 75.12 & 6.78 & 12.72 & -7.80 & -9.61 & 7.46 & 9.79 & -2.28 & 4.78 \\
\hline SBI Contra & -18.75 & -20.01 & 77.99 & 5.55 & 12.36 & -11.19 & -12.66 & 6.28 & 5.95 & 3.49 & 4.90 \\
\hline Reliance Equity 0pp & -18.63 & -23.43 & 91.92 & 19.76 & 24.71 & -7.88 & -6.53 & 9.08 & 13.79 & -0.64 & 10.22 \\
\hline Reliance Growth & -15.40 & -26.31 & 89.91 & 11.66 & 16.01 & -9.84 & -10.96 & 6.06 & 6.77 & -4.98 & 6.29 \\
\hline Reliance Regular Savings & -18.15 & -24.79 & 90.97 & 11.48 & 16.42 & -9.07 & -13.24 & 5.24 & 13.55 & -5.62 & 6.68 \\
\hline Reliance Tax Saver & -13.26 & -19.89 & 70.58 & 12.99 & 24.18 & -9.10 & -8.51 & 10.15 & 9.15 & -7.87 & 6.84 \\
\hline Reliance Vision Fund & -18.66 & -20.15 & 77.72 & 6.03 & 18.50 & -9.52 & -12.07 & 6.89 & 4.77 & -10.81 & 4.27 \\
\hline Principal Large Cap & -19.33 & -19.75 & 84.65 & 8.65 & 17.88 & -5.45 & -13.70 & 5.92 & 9.82 & -3.03 & 6.57 \\
\hline Birla Advantage & -19.74 & -27.67 & 90.49 & 5.03 & 15.13 & -9.53 & -11.81 & 2.04 & 6.92 & -2.92 & 4.79 \\
\hline Birla Equity & -21.05 & -26.65 & 85.67 & 10.17 & 15.39 & -11.00 & -12.43 & 4.00 & 9.47 & -2.44 & 5.11 \\
\hline Birla Frontline Equity & -16.10 & -20.89 & 80.64 & 9.31 & 15.63 & -4.13 & -11.27 & 4.58 & 10.97 & 2.69 & 7.14 \\
\hline HDFC Top 200 & -9.72 & -22.06 & 85.59 & 6.96 & 22.34 & -4.56 & -12.56 & 7.37 & 6.41 & -1.78 & 7.80 \\
\hline IDFC Classic Equity & -20.26 & -22.27 & 129.77 & 3.66 & 14.21 & -8.70 & -12.66 & 3.30 & 10.61 & 1.81 & 9.95 \\
\hline Tata Equity 0pp & -22.28 & -30.45 & 85.56 & 11.34 & 10.29 & -9.55 & -8.17 & 7.80 & 7.78 & -0.36 & 5.20 \\
\hline Sundaram Growth Fund & -16.15 & -36.65 & 89.84 & 2.04 & 20.85 & -9.82 & -13.97 & 8.03 & 6.56 & -5.59 & 4.51 \\
\hline Sundaram Tax Saver & -5.94 & -21.86 & 53.39 & 23.65 & -0.73 & -2.82 & 2.08 & -10.60 & 8.03 & 13.11 & 5.83 \\
\hline Principal Growth & -25.72 & -27.48 & 67.86 & 5.92 & 12.04 & -9.46 & -14.69 & 8.71 & 10.53 & -0.06 & 2.76 \\
\hline Principal Tax Savings & -32.53 & -32.62 & 68.07 & 6.65 & 12.46 & -9.55 & -14.15 & 8.98 & 11.06 & -0.03 & 1.83 \\
\hline IDFC Premier Equity & -16.22 & -23.01 & 85.10 & 22.62 & 19.62 & -11.01 & 2.77 & 3.01 & 10.96 & -0.11 & 9.37 \\
\hline Sundaram Select Midcap & -17.76 & -32.09 & 114.34 & 11.34 & 22.14 & -11.11 & -3.08 & 3.37 & 12.90 & -6.06 & 9.40 \\
\hline DSPBR Equity & -13.02 & -20.57 & 76.58 & 9.08 & 20.59 & -6.93 & -9.02 & 5.44 & 4.88 & -4.68 & 6.23 \\
\hline DSPBR Opp & -19.34 & -20.75 & 75.64 & 7.04 & 23.56 & -8.40 & -10.10 & 0.65 & 6.81 & 0.76 & 5.59 \\
\hline DSPBR Tax Saver & -17.82 & -24.20 & 79.36 & 10.97 & 19.13 & -9.20 & -11.99 & 6.11 & 10.55 & -0.07 & 6.28 \\
\hline Franklin India Prima & -22.15 & -31.53 & 98.10 & 17.10 & 17.41 & -11.14 & -6.04 & 7.28 & 9.80 & 3.08 & 8.19 \\
\hline Franklin India Taxshield & -13.89 & -22.21 & 68.36 & 13.63 & 16.23 & -2.00 & -6.04 & 6.72 & 6.39 & 0.68 & 6.79 \\
\hline HDFC Capital Builder & -16.59 & -26.45 & 84.42 & 13.10 & 21.22 & -5.18 & -10.99 & 6.14 & 7.97 & -0.99 & 7.27 \\
\hline HDFC Equity & -12.10 & -25.30 & 94.60 & 11.54 & 25.55 & -4.51 & -13.37 & 6.63 & 5.69 & -1.97 & 8.68 \\
\hline HDFC Tax Saver & -12.72 & -26.85 & 88.85 & 12.21 & 21.85 & -7.07 & -10.50 & 6.98 & 3.50 & -2.37 & 7.39 \\
\hline Sundaram Equity Mulitplier & -18.83 & -25.18 & 79.55 & 2.56 & 12.92 & -8.03 & -7.35 & 2.12 & 9.92 & -7.76 & 3.99 \\
\hline Principal Div Yield & -15.89 & -24.62 & 71.00 & 9.95 & 22.97 & -6.61 & -12.08 & 9.06 & 6.85 & -3.31 & 5.73 \\
\hline Birla Midcap & -22.91 & -31.02 & 118.27 & 15.25 & 12.83 & -12.31 & -6.90 & 6.35 & 6.86 & -3.46 & 8.30 \\
\hline DSPBR Small \& Midcap & -20.63 & -26.56 & 103.41 & 17.98 & 25.22 & -10.15 & -4.12 & 2.78 & 8.44 & -6.29 & 9.01 \\
\hline ICICI Discovery & -16.62 & -24.17 & 122.25 & 16.69 & 18.41 & -5.80 & -11.00 & 12.49 & 10.38 & 1.92 & 12.46 \\
\hline ICICI Midcap & -29.22 & -37.85 & 94.34 & 21.51 & 16.27 & -16.78 & -8.52 & 6.75 & 5.37 & -4.38 & 4.75 \\
\hline UTI Midcap & -18.14 & -30.54 & 103.44 & 12.97 & 20.59 & -11.28 & -2.73 & 1.57 & 9.51 & -6.01 & 7.94 \\
\hline Sundaram Smile & -16.68 & -30.27 & 119.87 & 5.49 & 19.17 & -17.06 & -7.82 & 2.34 & 18.44 & -18.20 & 7.53 \\
\hline ICICI Dynamic & -12.39 & -21.70 & 69.17 & 13.64 & 13.90 & 0.68 & -12.32 & 8.67 & 7.05 & -0.78 & 6.59 \\
\hline
\end{tabular}


Table 2 Continued

\begin{tabular}{|c|c|c|c|c|c|c|c|c|c|c|c|}
\hline \multirow{2}{*}{$\begin{array}{l}\text { Year } \rightarrow \\
\text { Fund } \downarrow\end{array}$} & \multicolumn{2}{|c|}{ 2008-09 } & \multicolumn{2}{|c|}{$2009-10$} & \multicolumn{2}{|c|}{$2010-11$} & \multicolumn{2}{|c|}{$2011-12$} & \multicolumn{2}{|c|}{$2012-13$} & \multirow[b]{2}{*}{ Mean } \\
\hline & $\mathrm{H} 1$ & $\mathrm{H} 2$ & $\mathrm{H} 1$ & $\mathrm{H} 2$ & $\mathrm{H} 1$ & $\mathrm{H} 2$ & $\mathrm{H} 1$ & $\mathrm{H} 2$ & $\mathrm{H} 1$ & $\mathrm{H} 2$ & \\
\hline SBI Magnum Equity & -19.61 & -22.88 & 78.23 & 8.90 & 16.77 & -5.21 & -10.59 & 8.54 & 6.58 & 1.96 & 6.27 \\
\hline Reliance Equity & -12.15 & -20.86 & 61.01 & -0.86 & 5.44 & -9.94 & -15.78 & 6.94 & 12.71 & -2.98 & 2.35 \\
\hline Franklin India Bluechip & -12.98 & -20.43 & 70.66 & 11.77 & 15.56 & -2.42 & -9.11 & 7.09 & 4.85 & 0.77 & 6.58 \\
\hline HDFC Growth & -12.25 & -28.98 & 76.79 & 8.82 & 25.12 & -5.66 & -9.69 & 7.58 & 6.19 & -3.40 & 6.45 \\
\hline HDFC Long Term Adv & -14.38 & -28.04 & 82.84 & 9.98 & 22.71 & -4.85 & -10.53 & 7.59 & 5.71 & 1.32 & 7.23 \\
\hline Tata PE Fund & -17.53 & -22.63 & 88.89 & 10.38 & 15.33 & -5.90 & -11.80 & 10.61 & 3.39 & -5.13 & 6.56 \\
\hline Tata Pure Equity & -17.05 & -19.65 & 68.76 & 10.57 & 18.24 & -10.33 & -7.70 & 6.21 & 9.79 & -1.24 & 5.76 \\
\hline Tata Tax Saving & -19.28 & -24.87 & 59.75 & -2.91 & 14.39 & -3.92 & -7.45 & -3.58 & 7.83 & -3.17 & 1.68 \\
\hline Fund Average & -17.33 & -24.76 & 83.46 & 10.08 & 17.38 & -7.87 & -9.62 & 5.63 & 8.29 & -2.30 & 6.29 \\
\hline \multicolumn{12}{|l|}{ Index Fund } \\
\hline BSE 100 & -19.33 & -26.14 & 80.68 & 4.14 & 14.27 & -5.00 & -14.68 & 6.40 & 7.27 & -0.40 & 4.72 \\
\hline BSE 200 & -20.06 & -26.69 & 83.70 & 4.99 & 15.05 & -6.00 & -14.73 & 6.39 & 6.94 & -0.85 & 4.87 \\
\hline BSE 500 & -20.97 & -28.06 & 85.97 & 5.60 & 15.39 & -6.85 & -14.14 & 5.85 & 6.61 & -1.69 & 4.77 \\
\hline BSE Midcap & -25.49 & -38.39 & 113.93 & 7.62 & 18.78 & -14.98 & -10.82 & 3.54 & 4.11 & -7.04 & 5.13 \\
\hline CNX 500 & -20.76 & -24.97 & 79.47 & 4.72 & 14.19 & -6.06 & -14.01 & 6.12 & 6.69 & -1.47 & 4.39 \\
\hline CNX Midcap & -22.23 & -30.33 & 97.02 & 14.77 & 18.94 & -12.27 & -11.77 & 8.70 & 1.67 & -5.60 & 5.89 \\
\hline CNX Nifty & -17.20 & -22.96 & 68.29 & 3.25 & 14.88 & -3.25 & -15.26 & 7.13 & 7.70 & -0.36 & 4.22 \\
\hline SENSEX & -18.46 & -24.51 & 76.41 & 2.34 & 14.50 & -3.11 & -15.38 & 5.78 & 7.81 & 0.39 & 4.58 \\
\hline
\end{tabular}

Table 3. Annual returns (in \%) and five yearly CAGR of sample and index funds

\begin{tabular}{llrrrrr}
\hline \multicolumn{1}{c}{ Fund $\downarrow$ Year $\rightarrow$} & $2008-09$ & $2009-10$ & $2010-11$ & $2011-12$ & $2012-13$ & Mean \\
\hline DSPBR Tiger & -39.04 & 79.04 & -0.23 & -9.70 & -1.81 & 5.65 \\
DSPBR Top 100 & -26.41 & 75.60 & 10.99 & -2.09 & 2.64 & 12.15 \\
UTI Dividend Yield & -22.36 & 87.05 & 16.03 & -3.35 & 1.64 & 15.80 \\
UTI Equity & -30.74 & 82.66 & 14.87 & -1.69 & 8.07 & 14.63 \\
SBI Magnum Tax Gain & -39.48 & 86.99 & 3.93 & -2.86 & 7.28 & 11.17 \\
SBI Contra & -35.01 & 87.87 & -0.22 & -7.17 & 9.64 & 11.02 \\
Reliance Equity 0pp & -37.69 & 129.84 & 14.89 & 1.95 & 13.07 & 24.41 \\
Reliance Growth & -37.65 & 112.06 & 4.59 & -5.56 & 1.45 & 14.98 \\
Reliance Regular Savings & -38.44 & 112.90 & 5.87 & -8.70 & 7.17 & 15.76 \\
Reliance Tax Saver & -30.52 & 92.75 & 12.88 & 0.78 & 0.56 & 15.29 \\
Reliance Vision Fund & -35.05 & 88.44 & 7.22 & -6.01 & -6.56 & 9.61 \\
Principal Large Cap & -35.26 & 100.62 & 11.46 & -8.59 & 6.49 & 14.94 \\
Birla Advantage & -41.95 & 100.08 & 4.15 & -10.01 & 3.81 & 11.22 \\
Birla Equity & -42.09 & 104.55 & 2.70 & -8.92 & 6.80 & 12.61 \\
Birla Frontline Equity & -33.63 & 97.46 & 10.85 & -7.21 & 13.95 & 16.29 \\
HDFC Top 200 & -29.64 & 98.51 & 16.76 & -6.12 & 4.52 & 16.80 \\
IDFC Classic Equity & -38.01 & 138.18 & 4.27 & -9.78 & 12.61 & 21.45 \\
Tata Equity Opp & -45.94 & 106.60 & -0.25 & -1.01 & 7.39 & 13.36 \\
Sundaram Growth Fund & -46.89 & 93.70 & 8.99 & -7.06 & 0.60 & 9.87 \\
Sundaram Tax Saver & -26.50 & 89.67 & -3.53 & -8.74 & 22.19 & 14.62 \\
Principal Growth & -46.13 & 77.79 & 1.44 & -7.26 & 10.47 & 7.26 \\
Principal Tax Savings & -54.54 & 79.25 & 1.72 & -6.44 & 11.03 & 6.20 \\
IDFC Premier Equity & -35.50 & 126.97 & 6.45 & 5.86 & 10.84 & 22.92 \\
Sundaram Select Midcap & -44.14 & 138.65 & 8.57 & 0.19 & 6.06 & 21.86 \\
DSPBR Equity & -30.91 & 92.62 & 12.24 & -4.07 & -0.04 & 13.97 \\
DSPBR Opp & -36.08 & 88.00 & 13.18 & -9.52 & 7.61 & 12.64 \\
DSPBR Tax Saver & -37.71 & 99.03 & 8.17 & -6.61 & 10.47 & 14.67 \\
\hline
\end{tabular}


Table 3 Continued

\begin{tabular}{|c|c|c|c|c|c|c|}
\hline Fund $\downarrow$ & 2008-09 & $2009-10$ & $2010-11$ & $2011-12$ & $2012-13$ & Mean \\
\hline Franklin India Prima & -46.69 & 131.98 & 4.33 & 0.80 & 13.18 & 20.72 \\
\hline Franklin India Taxshield & -33.02 & 91.30 & 13.91 & 0.28 & 7.12 & 15.92 \\
\hline HDFC Capital Builder & -38.65 & 108.59 & 14.94 & -5.53 & 6.90 & 17.25 \\
\hline HDFC Equity & -34.34 & 117.06 & 19.89 & -7.63 & 3.61 & 19.72 \\
\hline HDFC Tax Saver & -36.15 & 111.90 & 13.24 & -4.26 & 1.05 & 17.16 \\
\hline Sundaram Equity Mulitplier & -39.27 & 84.14 & 3.85 & -5.38 & 1.38 & 8.94 \\
\hline Principal Div Yield & -36.60 & 88.01 & 14.84 & -4.11 & 3.31 & 13.09 \\
\hline Birla Midcap & -46.82 & 151.55 & -1.06 & -1.00 & 3.16 & 21.17 \\
\hline DSPBR Small \& Midcap & -41.72 & 139.99 & 12.51 & -1.46 & 1.62 & 22.19 \\
\hline ICICI Discovery & -36.77 & 159.35 & 11.55 & 0.12 & 12.50 & 29.35 \\
\hline ICICI Midcap & -56.01 & 136.15 & -3.24 & -2.35 & 0.76 & 15.06 \\
\hline UTI Midcap & -43.14 & 129.82 & 6.99 & -1.20 & 2.93 & 19.08 \\
\hline Sundaram Smile & -41.90 & 131.93 & -1.16 & -5.66 & -3.12 & 16.02 \\
\hline ICICI Dynamic & -31.40 & 92.24 & 14.67 & -4.72 & 6.21 & 15.40 \\
\hline Sundaram Select Focus & -39.80 & 77.97 & 5.92 & -11.24 & 5.70 & 7.71 \\
\hline SBI Magnum Equity & -38.00 & 94.09 & 10.69 & -2.95 & 8.66 & 14.50 \\
\hline Reliance Equity & -30.48 & 59.63 & -5.04 & -9.94 & 9.35 & 4.70 \\
\hline Franklin India Bluechip & -30.76 & 90.74 & 12.77 & -2.67 & 5.66 & 15.15 \\
\hline HDFC Growth & -37.68 & 92.38 & 18.04 & -2.85 & 2.59 & 14.50 \\
\hline HDFC Long Term Adv & -38.39 & 101.09 & 16.76 & -3.75 & 7.10 & 16.56 \\
\hline Tata PE Fund & -36.19 & 108.49 & 8.53 & -2.44 & -1.91 & 15.30 \\
\hline Tata Pure Equity & -33.35 & 86.60 & 6.03 & -1.97 & 8.42 & 13.15 \\
\hline Tata Tax Saving & -39.36 & 55.10 & 9.91 & -10.76 & 4.41 & 3.86 \\
\hline Fund Average & -37.68 & 102.14 & 8.14 & -4.61 & 5.77 & 14.75 \\
\hline \multicolumn{7}{|l|}{ Index Fund } \\
\hline BSE 100 & -40.42 & 88.17 & 8.55 & -9.23 & 6.84 & 10.78 \\
\hline BSE 200 & -41.40 & 92.87 & 8.15 & -9.28 & 6.03 & 11.27 \\
\hline BSE 500 & -43.14 & 96.38 & 7.48 & -9.11 & 4.81 & 11.28 \\
\hline BSE Midcap & -54.09 & 130.23 & 0.99 & -7.67 & -3.22 & 13.25 \\
\hline CNX 500 & -40.55 & 87.95 & 7.26 & -8.75 & 5.13 & 10.21 \\
\hline CNX Midcap & -45.82 & 126.12 & 4.35 & -4.09 & -4.02 & 15.31 \\
\hline CNX Nifty & -36.21 & 73.76 & 11.14 & -9.23 & 7.31 & 9.35 \\
\hline SENSEX & -38.44 & 80.54 & 10.94 & -10.50 & 8.23 & 10.15 \\
\hline
\end{tabular}

in Table 4. As we observed in Tables 1, 2 and 3 earlier, exceptionally higher returns caused two year rolling CAGR (Table 4) of all the funds be the highest across all CAGR. To be specific as far as two year rolling CAGR is concerned ICICI Discovery fund, DSPBR Small \& Midcap fund and Reliance Equity Opportunity have topped the list with 70.09 CAGR, 64.32 CAGR and 62.5 CAGR respectively. CAGR of all rolling frequencies also reveal that ICICI Discovery fund, Reliance Equity Opportunity fund and DSPBR Small \& Midcap fund have exhibited consistent performance across all rolling periods. These funds could sustain the volatility in the market and perform better. This in turn implies that the investment in these funds have earned better returns on any frequency basis during the observed period. Barring a few funds, majority of the funds have generated negative CAGR in two yearly returns during 2010-12. If we compare five yearly CAGR with that of two yearly and three yearly, we notice that the CAGR is regressed over a period of time (i.e. 2008-13) implying that the investors could not make any exceptional returns if the investment held for such long period of time. This observation goes in line with the hypothesis that no investor could make abnormal returns in the long-run. Among the benchmark funds it is only in case of CNX Nifty that CAGR of returns was positive except during 2011-13. Even on five yearly CAGR basis this benchmark fund performed better than other benchmark funds and topped the list.

Apart from analyzing the returns on the basis of various frequencies, we also have computed some of the key statistical measures to understand the risk element in the returns. Some of such measures we have are Standard Deviation (S.D), beta co-efficient ( $\beta$ ), multiple ' $R$ ' and $R^{2}$. It may be noted that beta co-efficient, 
multiple ' $R$ ' and $R^{2}$ (Table 5) are arrived at by running regression analysis between fund's return and the return of the concerned benchmark fund for the years 2008-09 through 2012-12. The values are summarized in Table 6. As we observe, the beta co-efficient of the majority of the funds was more than 1 in the year 2012-13 indicating very high sensitivity of the funds return to that of benchmark. It is interesting to note that the beta of Sundaram Tax Saver fund was negative in the years 2008-09, 2009-10 and 2012-13 while no other fund had negative beta any time during the study period. Comparison of annual betas with the annual returns (in Table 3 ) indicates a bit contrasting observation. In the sense that the returns in the year 2009-10 were exceptionally high but the beta values (Table 6) of the same were not very high. This observation is true in case of almost all sample funds. This implies that the funds generated higher returns for lower risk when compared to the return-risk relationship in the rest of the years. However the standard deviation in the year 2009-10 was found to be higher in case of most of the funds. The performance of the majority of the funds was almost in line with that of the benchmark during all the years barring 2012-13. There is no fund with zero betas implying that the performance of every fund depended on market's performance. This is also supported by multiple ' $R$ '. The values of multiple ' $R$ ' reveal that there existed very strong significant correlation between the return of the funds and that of benchmark. The values of ' $R$ ' across all the funds and all the years were very close to 1 . This reveals very high dependency of funds performance on that of benchmark. Even the ' $\mathrm{R}^{2}$ ' values of almost all the funds are found to be very close to 1 during the study period. This also implies that the variability in the returns of the sample funds was largely explained by the market performance. This means that the explained portion of the total risk in the returns of the sample funds was very high, almost close to 1 . This in turn implies that the fund managers seem to have diversified non-systematic to the fullest extent making their portfolios almost fully diversified in line with the market.

Having discussed the performance of the funds, in terms of only the return without adjusting it for the risk, we now move on to discussing the risk-adjusted performance of sample funds. As we mentioned earlier we discuss this measure-wise that we chose.

\subsection{Sharpe Measure}

As suggested by Sharpe, the yearly return of the fund has been adjusted to total risk measured in terms of standard deviation and the results are summarized in Table 6 . Higher the Sharpe ratio, better is the performance of the fund and vice versa. The fund's performance is also ranked and mentioned next to Sharpe ratio for every fund during the study period. Like in the case of annual fund returns (in Table 3), the Sharpe Ratio of all the funds in 2008-09 and 2011-12 is found to be negative. This is of course obvious because the excess return of the fund over risk free return is adjusted to the standard deviation and the standard deviation can never be negative. This is in fact one of the serious limitations of Sharpe measure. Out of five years of the study period, almost all the funds have performed extremely well in the year 2009-10. This observation is same like

Table 4. Rolling returns (in \%) of sample and index funds

\begin{tabular}{|c|c|c|c|c|c|c|c|c|}
\hline \multirow[b]{2}{*}{ Fund $\downarrow$} & \multicolumn{4}{|c|}{2 Year Rolling CAGR } & \multicolumn{3}{|c|}{3 Year Rolling CAGR } & \multirow{2}{*}{$\begin{array}{c}5 \text { Year CAGR } \\
2008-2013\end{array}$} \\
\hline & 2008-10 & 2009-11 & 2010-12 & 2011-13 & 2008-11 & 2009-12 & 2010-13 & \\
\hline DSPBR Tiger & 4.47 & 33.65 & -5.09 & -5.84 & 2.88 & 17.27 & -4.01 & -0.70 \\
\hline DSPBR Top 100 & 13.68 & 39.61 & 4.24 & 0.25 & 12.77 & 24.03 & 3.71 & 7.59 \\
\hline UTI Dividend Yield & 20.51 & 47.32 & 5.90 & -0.88 & 19.00 & 28.01 & 4.46 & 10.61 \\
\hline UTI Equity & 12.47 & 44.85 & 6.27 & 3.07 & 13.27 & 27.30 & 6.86 & 9.07 \\
\hline SBI Magnum Tax Gain & 6.39 & 39.41 & 0.47 & 2.08 & 5.56 & 23.59 & 2.69 & 4.16 \\
\hline SBI Contra & 10.50 & 36.92 & -3.76 & 0.88 & 6.81 & 20.28 & 0.52 & 4.40 \\
\hline Reliance Equity 0pp & 19.67 & 62.50 & 8.23 & 7.36 & 18.05 & 39.11 & 9.82 & 13.66 \\
\hline
\end{tabular}


Table 4 Continued

\begin{tabular}{|c|c|c|c|c|c|c|c|c|}
\hline \multirow[b]{2}{*}{ Fund $\downarrow$} & \multicolumn{4}{|c|}{2 Year Rolling CAGR } & \multicolumn{3}{|c|}{3 Year Rolling CAGR } & \multirow{2}{*}{$\frac{5 \text { Year CAGR }}{2008-2013}$} \\
\hline & $2008-10$ & $2009-11$ & $2010-12$ & $2011-13$ & $2008-11$ & $2009-12$ & $2010-13$ & \\
\hline Reliance Regular Savings & 14.48 & 50.13 & -1.68 & -1.08 & 11.54 & 27.20 & 1.18 & 6.31 \\
\hline Reliance Tax Saver & 15.73 & 47.50 & 6.66 & 0.67 & 14.77 & 29.91 & 4.59 & 8.91 \\
\hline Reliance Vision Fund & 10.63 & 42.14 & 0.39 & -6.28 & 9.48 & 23.83 & -1.98 & 2.88 \\
\hline Principal Large Cap & 13.96 & 49.54 & 0.94 & -1.34 & 13.12 & 26.91 & 2.76 & 7.10 \\
\hline Birla Advantage & 7.77 & 44.35 & -3.19 & -3.35 & 6.55 & 23.31 & -0.91 & 2.48 \\
\hline Birla Equity & 8.84 & 44.94 & -3.29 & -1.38 & 6.75 & 24.14 & -0.04 & 3.42 \\
\hline Birla Frontline Equity & 14.48 & 47.94 & 1.42 & 2.83 & 13.26 & 26.64 & 5.44 & 8.97 \\
\hline HDFC Top 200 & 18.18 & 52.24 & 4.70 & -0.94 & 17.70 & 29.58 & 4.64 & 9.86 \\
\hline IDFC Classic Equity & 21.51 & 57.59 & -3.01 & 0.79 & 15.47 & 30.85 & 1.94 & 9.36 \\
\hline Tata Equity 0pp & 5.68 & 43.56 & -0.63 & 3.11 & 3.67 & 26.83 & 1.98 & 3.44 \\
\hline Sundaram Growth Fund & 1.43 & 45.30 & 0.64 & -3.31 & 3.89 & 25.19 & 0.63 & 0.95 \\
\hline Principal Growth & -2.14 & 34.29 & -3.01 & 1.22 & -0.96 & 18.70 & 1.29 & -0.09 \\
\hline Principal Tax Savings & -9.73 & 35.03 & -2.45 & 1.92 & -6.06 & 19.49 & 1.85 & -2.95 \\
\hline IDFC Premier Equity & 21.00 & 55.44 & 6.15 & 8.32 & 15.94 & 36.76 & 7.69 & 12.83 \\
\hline Sundaram Select Midcap & 15.45 & 60.97 & 4.29 & 3.08 & 13.11 & 37.43 & 4.88 & 8.99 \\
\hline DSPBR Equity & 15.36 & 47.03 & 3.76 & -2.08 & 14.31 & 27.52 & 2.48 & 7.45 \\
\hline DSPBR 0pp & 9.62 & 45.87 & 1.20 & -1.32 & 10.80 & 24.40 & 3.29 & 5.78 \\
\hline DSPBR Tax Saver & 11.35 & 46.73 & 0.51 & 1.57 & 10.28 & 26.21 & 3.72 & 6.71 \\
\hline Franklin India Prima & 11.20 & 55.57 & 2.55 & 6.81 & 8.86 & 34.62 & 5.98 & 8.04 \\
\hline Franklin India Taxshield & 13.20 & 47.62 & 6.88 & 3.64 & 13.43 & 29.77 & 6.96 & 9.41 \\
\hline HDFC Capital Builder & 13.12 & 54.84 & 4.20 & 0.49 & 13.73 & 31.33 & 5.10 & 8.24 \\
\hline HDFC Equity & 19.38 & 61.32 & 5.24 & -2.17 & 19.55 & 33.96 & 4.69 & 10.34 \\
\hline HDFC Tax Saver & 16.32 & 54.90 & 4.12 & -1.64 & 15.28 & 31.95 & 3.09 & 8.19 \\
\hline Sundaram Equity Mulitplier & 5.75 & 38.29 & -0.87 & -2.06 & 5.11 & 21.86 & -0.13 & 2.18 \\
\hline Principal Div Yield & 9.18 & 46.94 & 4.94 & -0.47 & 11.03 & 27.45 & 4.39 & 6.28 \\
\hline Birla Midcap & 15.66 & 57.76 & -1.03 & 1.06 & 9.80 & 35.07 & 0.35 & 6.21 \\
\hline DSPBR Small \& Midcap & 18.27 & 64.32 & 5.29 & 0.07 & 16.32 & 38.57 & 4.05 & 9.52 \\
\hline ICICI Discovery & 28.06 & 70.09 & 5.68 & 6.13 & 22.30 & 42.55 & 7.91 & 15.56 \\
\hline ICICI Midcap & 1.92 & 51.16 & -2.79 & -0.81 & 0.17 & 30.68 & -1.62 & -0.22 \\
\hline UTI Midcap & 14.32 & 56.81 & 2.81 & 0.84 & 11.82 & 34.43 & 2.85 & 7.29 \\
\hline Sundaram Smile & 16.08 & 51.41 & -3.44 & -4.40 & 10.02 & 29.32 & -3.33 & 4.01 \\
\hline ICICI Dynamic & 14.84 & 48.47 & 4.53 & 0.60 & 14.78 & 28.07 & 5.09 & 8.88 \\
\hline Sundaram Select Focus & 3.51 & 37.30 & -3.04 & -3.14 & 4.31 & 18.72 & -0.21 & 1.26 \\
\hline SBI Magnum Equity & 9.70 & 46.57 & 3.64 & 2.69 & 10.03 & 27.75 & 5.29 & 7.03 \\
\hline Reliance Equity & 5.35 & 23.12 & -7.52 & -0.76 & 1.76 & 10.93 & -2.21 & 0.75 \\
\hline Franklin India Bluechip & 14.92 & 46.66 & 4.77 & 1.41 & 14.20 & 27.93 & 5.06 & 8.90 \\
\hline HDFC Growth & 9.49 & 50.69 & 7.09 & -0.17 & 12.27 & 30.18 & 5.57 & 7.12 \\
\hline HDFC Long Term Adv & 11.31 & 53.23 & 6.01 & 1.53 & 13.09 & 31.23 & 6.37 & 8.32 \\
\hline Tata PE Fund & 15.34 & 50.42 & 2.90 & -2.18 & 13.02 & 30.21 & 1.27 & 6.68 \\
\hline Tata Pure Equity & 11.52 & 40.66 & 1.95 & 3.09 & 9.66 & 24.71 & 4.06 & 6.98 \\
\hline Tata Tax Saving & -3.01 & 30.56 & -0.97 & -3.47 & 1.11 & 15.01 & 0.79 & -0.75 \\
\hline \multicolumn{9}{|l|}{ Index Fund } \\
\hline BSE 100 & 5.89 & 42.92 & -0.73 & -1.52 & 6.77 & 22.85 & 1.73 & 3.37 \\
\hline BSE 200 & 6.31 & 44.42 & -0.95 & -1.93 & 6.92 & 23.69 & 1.32 & 3.29 \\
\hline BSE 500 & 5.67 & 45.28 & -1.16 & -2.40 & 6.27 & 24.26 & 0.79 & 2.71 \\
\hline BSE Midcap & 2.81 & 52.48 & -3.44 & -5.47 & 2.20 & 29.00 & -3.36 & -0.94 \\
\hline CNX 500 & 5.71 & 41.99 & -1.07 & -2.05 & 6.22 & 22.53 & 0.96 & 2.83 \\
\hline CNX Midcap & 10.69 & 53.61 & 0.04 & -4.05 & 8.53 & 31.29 & -1.33 & 3.31 \\
\hline CNX Nifty & 5.28 & 38.96 & 0.44 & -1.30 & 7.20 & 20.57 & 2.68 & 3.71 \\
\hline SENSEX & 5.42 & 41.52 & -0.35 & -1.58 & 7.23 & 21.48 & 2.43 & 3.61 \\
\hline
\end{tabular}




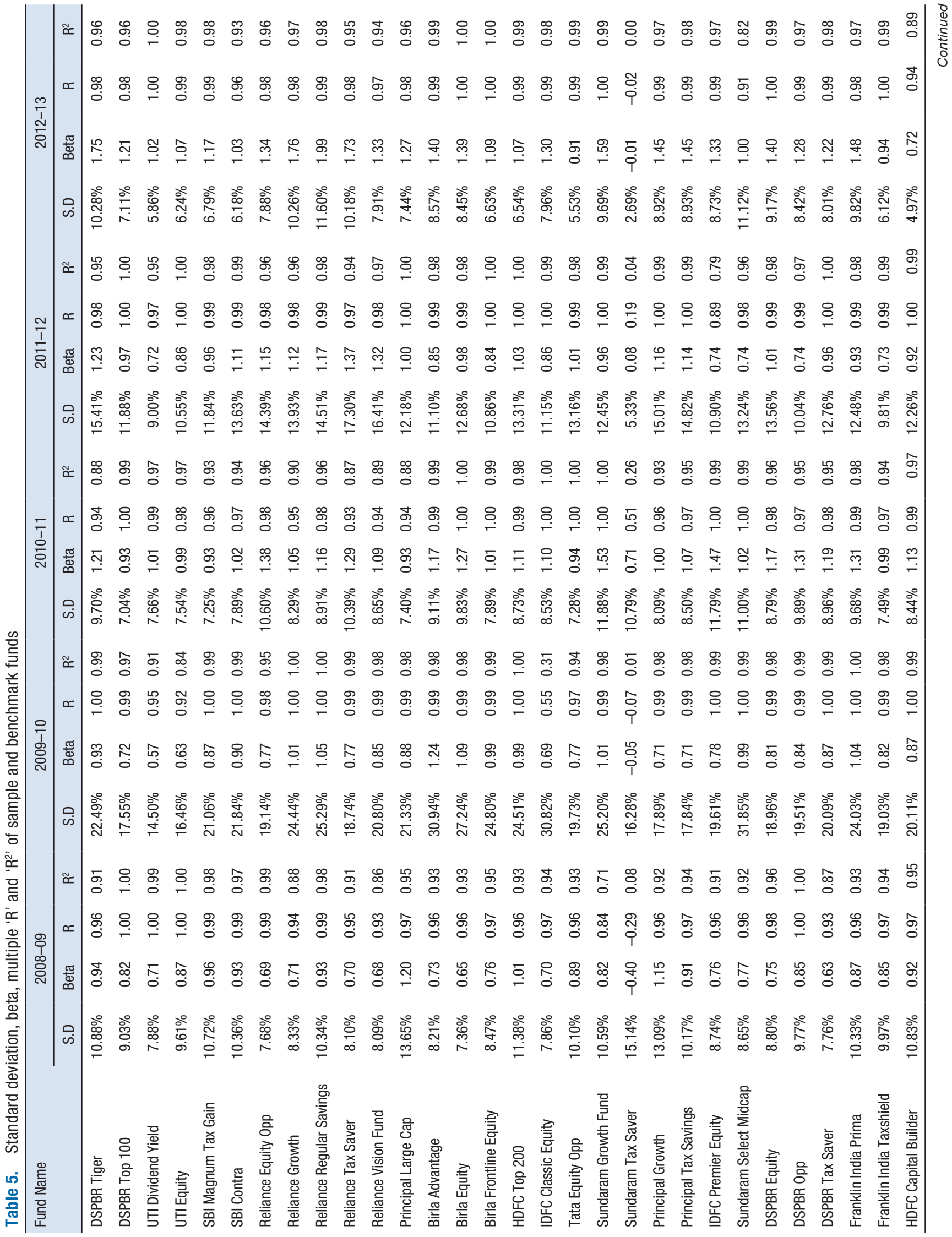




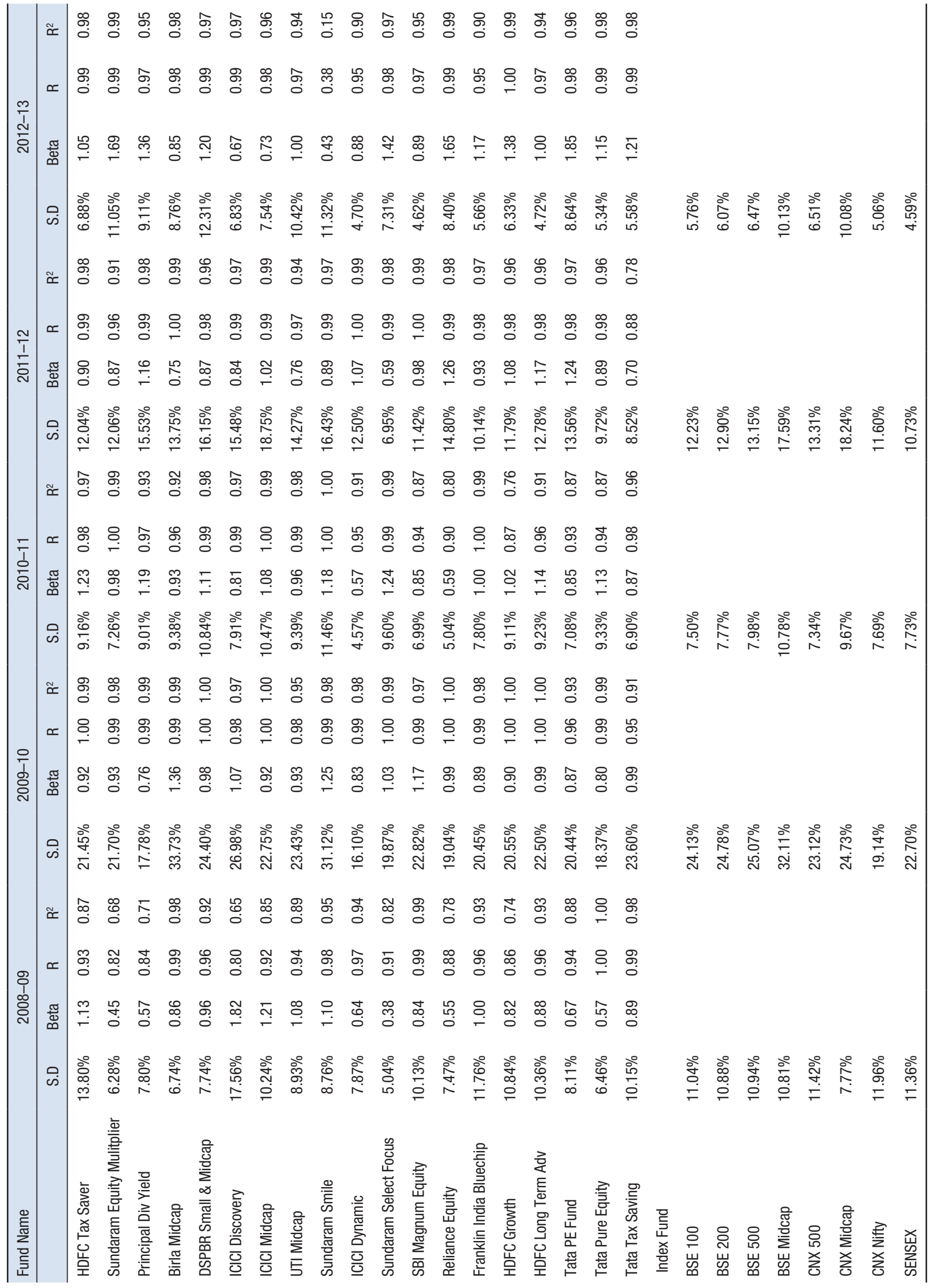


Table 6. Risk-adjusted performance and rank of sample funds according to Sharpe Ratio (S.R)

\begin{tabular}{|c|c|c|c|c|c|c|c|c|c|c|}
\hline Year $\rightarrow$ & & & & & & & & & & -13 \\
\hline Fund Name & S.R & Rank & S.R & Rank & S.R & Rank & S.R & Rank & S.R & Rank \\
\hline Birla Advantage & -5.97 & 42 & 3.10 & 48 & -0.23 & 36 & -1.66 & 46 & -0.01 & 35 \\
\hline Birla Equity & -6.68 & 47 & 3.68 & 44 & -0.36 & 40 & -1.36 & 42 & 0.02 & 20 \\
\hline Birla Frontline Equity & -4.81 & 25 & 3.76 & 42 & 0.59 & 21 & -1.43 & 44 & 0.08 & 6 \\
\hline Birla Midcap & -8.01 & 49 & 4.37 & 25 & -0.78 & 45 & -0.68 & 10 & 0.05 & 14 \\
\hline DSPBR Equity & -4.32 & 17 & 4.66 & 19 & 0.69 & 16 & -0.92 & 18 & -0.04 & 41 \\
\hline DSPBR Opp & -4.42 & 21 & 4.29 & 29 & 0.70 & 15 & -1.78 & 47 & 0.03 & 15 \\
\hline DSPBR Small \& Midcap & -6.31 & 46 & 5.56 & 6 & 0.58 & 22 & -0.61 & 7 & 0.08 & 5 \\
\hline DSPBR Tax Saver & -5.78 & 39 & 4.72 & 17 & 0.22 & 26 & -1.17 & 37 & 0.06 & 11 \\
\hline DSPBR Tiger & -4.24 & 16 & 3.33 & 47 & -0.67 & 44 & -1.17 & 36 & -0.08 & 48 \\
\hline DSPBR Top 100 & -3.71 & 7 & 4.07 & 36 & 0.68 & 17 & -0.88 & 17 & -0.04 & 39 \\
\hline Franklin India Bluechip & -3.22 & 5 & 4.23 & 31 & 0.84 & 11 & -1.09 & 32 & -0.03 & 37 \\
\hline Franklin India Prima & -5.21 & 30 & 5.32 & 9 & -0.20 & 34 & -0.61 & 6 & 0.10 & 3 \\
\hline Franklin India Taxshield & -4.03 & 11 & 4.58 & 20 & 1.03 & 9 & -0.82 & 14 & 0.02 & 18 \\
\hline HDFC Capital Builder & -4.23 & 15 & 5.19 & 12 & 1.03 & 8 & -1.13 & 33 & 0.01 & 25 \\
\hline HDFC Equity & -3.77 & 9 & 4.48 & 23 & 1.43 & 2 & -1.08 & 30 & -0.01 & 33 \\
\hline HDFC Growth & -4.13 & 14 & 4.29 & 30 & 1.30 & 3 & -0.95 & 21 & -0.06 & 46 \\
\hline HDFC Long Term Adv & -4.39 & 19 & 4.31 & 27 & 1.14 & 7 & -0.95 & 19 & -0.01 & 30 \\
\hline HDFC Tax Saver & -3.14 & 4 & 5.02 & 14 & 0.77 & 13 & -1.05 & 28 & -0.04 & 40 \\
\hline HDFC Top 200 & -3.23 & 6 & 3.85 & 40 & 1.21 & 5 & -1.09 & 31 & -0.01 & 34 \\
\hline ICICI Discovery & -2.50 & 2 & 5.75 & 4 & 0.67 & 18 & -0.53 & 4 & 0.12 & 2 \\
\hline ICICI Dynamic & -4.90 & 27 & 5.47 & 7 & 1.85 & 1 & -1.05 & 27 & -0.01 & 31 \\
\hline ICICI Midcap & -6.17 & 44 & 5.80 & 3 & -0.90 & 48 & -0.57 & 5 & 0.02 & 21 \\
\hline IDFC Classic Equity & -5.74 & 38 & 4.35 & 26 & -0.23 & 37 & -1.63 & 45 & 0.07 & 7 \\
\hline IDFC Premier Equity & -4.88 & 26 & 6.26 & 2 & 0.02 & 30 & -0.23 & 1 & 0.07 & 8 \\
\hline Principal Div Yield & -5.60 & 36 & 4.71 & 18 & 0.96 & 10 & -0.80 & 13 & -0.01 & 29 \\
\hline Principal Growth & -4.07 & 13 & 4.11 & 34 & -0.59 & 42 & -1.04 & 26 & 0.05 & 13 \\
\hline Principal Large Cap & -3.11 & 3 & 4.52 & 21 & 0.71 & 14 & -1.39 & 43 & 0.00 & 28 \\
\hline Principal Tax Savings & -6.06 & 43 & 4.20 & 33 & -0.53 & 41 & -1.00 & 24 & 0.06 & 12 \\
\hline Reliance Equity & -5.03 & 28 & 2.91 & 49 & -2.23 & 50 & -1.24 & 39 & 0.03 & 17 \\
\hline Reliance Equity 0pp & -5.84 & 40 & 6.56 & 1 & 0.82 & 12 & -0.45 & 3 & 0.07 & 10 \\
\hline Reliance Growth & -5.37 & 34 & 4.41 & 24 & -0.20 & 35 & -1.00 & 25 & -0.04 & 43 \\
\hline Reliance Regular Savings & -4.41 & 20 & 4.30 & 28 & -0.04 & 33 & -1.18 & 38 & 0.02 & 19 \\
\hline Reliance Tax Saver & -4.65 & 24 & 4.72 & 16 & 0.64 & 19 & -0.44 & 2 & -0.05 & 45 \\
\hline Reliance Vision Fund & -5.21 & 31 & 4.05 & 37 & 0.12 & 28 & -0.88 & 16 & -0.13 & 50 \\
\hline SBI Contra & -4.07 & 12 & 3.83 & 41 & -0.82 & 46 & -1.14 & 35 & 0.03 & 16 \\
\hline SBI Magnum Equity & -4.45 & 22 & 3.94 & 38 & 0.64 & 20 & -0.99 & 23 & 0.01 & 23 \\
\hline SBI Magnum Tax Gain & -4.35 & 18 & 3.93 & 39 & -0.32 & 38 & -0.95 & 20 & 0.01 & 26 \\
\hline Sundaram Equity Mulitplier & -7.39 & 48 & 3.68 & 45 & -0.33 & 39 & -1.14 & 34 & -0.02 & 36 \\
\hline Sundaram Growth Fund & -5.10 & 29 & 3.55 & 46 & 0.23 & 25 & -1.24 & 40 & -0.04 & 42 \\
\hline Sundaram Select Focus & -9.30 & 50 & 3.71 & 43 & -0.03 & 32 & -2.82 & 49 & -0.01 & 32 \\
\hline Sundaram Select Midcap & -5.93 & 41 & 4.22 & 32 & 0.21 & 27 & -0.62 & 8 & 0.09 & 4 \\
\hline Sundaram Smile & -5.60 & 35 & 4.10 & 35 & -0.64 & 43 & -0.85 & 15 & -0.06 & 47 \\
\hline Sundaram Tax Saver & -2.22 & 1 & 5.25 & 10 & -0.90 & 49 & -3.21 & 50 & 0.14 & 1 \\
\hline Tata Equity 0pp & -5.25 & 32 & 5.19 & 11 & -0.89 & 47 & -0.71 & 11 & 0.01 & 24 \\
\hline Tata PE Fund & -5.34 & 33 & 5.10 & 13 & 0.33 & 24 & -0.80 & 12 & -0.10 & 49 \\
\hline Tata Pure Equity & -6.27 & 45 & 4.49 & 22 & -0.02 & 31 & -1.06 & 29 & 0.00 & 27 \\
\hline Tata Tax Saving & -4.58 & 23 & 2.16 & 50 & 0.53 & 23 & -2.25 & 48 & -0.04 & 38 \\
\hline UTI Dividend Yield & -3.74 & 8 & 5.71 & 5 & 1.28 & 4 & -1.30 & 41 & -0.05 & 44 \\
\hline UTI Equity & -3.94 & 10 & 4.76 & 15 & 1.15 & 6 & -0.95 & 22 & 0.01 & 22 \\
\hline UTI Midcap & -5.63 & 37 & 5.36 & 8 & 0.08 & 29 & -0.67 & 9 & 0.07 & 9 \\
\hline
\end{tabular}


that of quarterly, half yearly and yearly performance even without risk consideration. While there is no fund that maintained consistent Sharpe ratio and rank, a few funds have exhibited such behavior. For instance, ICICI Discovery fund maintained consistent rank 2, rank 4, rank 18 (an exception), rank 4 and rank 2 during five years respectively. If we recollect our discussions earlier, we observe that this fund has been found to have performed consistently. Followed by this DSPBR Equity fund also maintained such kind of consistency with an exception in the last year. The performance of a few funds (like that of ICICI Midcap) showed the improvement and decline year on year basis. Thus the values in Table 6 reveal that the performance of a given fund according to Sharpe measure varied over the years. Most of the funds performed better in some years and bad in other years. Also this is evidenced by the rank in every year. Having run ANOVA to test $\mathrm{HO}_{2}$, we find observed 'p' value (0.00) being less than critic ' $p$ ' value $(0.05)$ and therefore reject the hypothesis and conclude that the performance of sample funds, according to Sharpe measure varied during the years 2008-09 through 2012-13. This implies that the time factor played a role in the performance of a fund.

\subsection{Treynor Ratio}

While Treynor also considers excess return of the fund to measure its performance, unlike Sharpe, he considers adjusting such excess return to systematic risk only. Treynor ratio and the rank accordingly of the sample funds have been captured in Table 7. Treynor ratio of all the funds in the years 2008-09 and 2011-12 tended to be negative implying negative performance even according to this measure. ICICI Discovery fund, like in case of Sharpe measure, sustained the consistency in its performance. In fact it ranked excellent throughout the period. Sundaram Tax Saver fund performed very badly in all the years with an exception in the first year. It ranked as the top fund in the first year but as the last fund in the rest of the years. Reliance Equity Opportunity fund also has done very well throughout, again, with an exception in the first year. Thus Treynor ratios in Table 7 reveal mixed performance of the funds during the observed period. In order to find out if the performance of the funds according to Treynor measure varied over the years, we have tested $\mathrm{HO}_{3}$. As the calculated 'p' value $(0.00)$ is less than the critical 'p' value $(0.05)$ we reject $\mathrm{HO}_{3}$ and conclude that the performance of the sample funds varied over the years 2008-09 through 2012-13. This implies that the time factor played a role performance of the funds even in case of Treynor ratio.

\subsection{Jensen's Alpha}

While Sharpe and Treynor adjust the excess return to total risk and systematic risk respectively, Jensen considers abnormal return (excess of realized return over CAPM based expected return) known as alpha. Higher the alpha value better is the performance of the fund and vice versa. The negative alphas indicate under performance of the fund when compared to the expected return. The alpha values of the sample funds have been depicted in Table 8. Similar to Sharpe ratio and Treynor ratio, alpha value of most of the funds in the year 2008-09 are found to be negative indicating that the funds in this year under performed when compared to their systematic risk-adjusted expected return. ICICI Discovery fund again posted positive alphas in all the years varying ranking. The performance of UTI Dividend Yield was among the top ten funds during the first three years but dropped drastically in the last two years. It is found that UTI Equity fund also has performed consistently during the first three years but slightly lower in the last two years. When compared to the rest of the funds, this fund has done better on an aggregate basis. The alpha values and ranks in Table 8 reveal that performance of the most of the sample funds varied over the years. This observation is also proven through hypothesis testing. As the observed ' $\mathrm{p}$ ' value $(0.00)$ with regard to $\mathrm{HO}_{4}$, is less than the critical ' $p$ ' value we reject $\mathrm{HO}_{4}$ and conclude that the performance of the sample funds according to Jensen's alpha varied over the years 2008-09 through 2012-13. This in turn implies that the time factor played a role in the performance, as measured by Jensen's alpha, of sample funds. Thus, even according to Jensen's alpha the performance of the most of the funds was not consistent.

\subsection{Information Ratio}

Information ratio indicates fund manager's ability to beat the benchmark. This is computed by dividing the 
excess return of fund (over that of benchmark) by the tracking error (standard deviation of the excess returns of fund over the benchmark return). Higher the ratio, better is the fund manager's performance and vice versa. The information ratios of sample funds are summarized in Table 9. Unlike the performance according to Sharpe, Treynor and Jensen, the performance of the most of the funds according to this ratio is positive in the years 2008-09. The ratio of four funds, DSPBR Top 100, UTI Dividend Yield, UTI Equity and Tata Pure Equity was very high in this year. This implies that these funds have performed extremely well in the first year while the same did not according other measures that we discussed so for. Interestingly the performance of ICICI Discovery fund was lower than that of many other funds where as it maintained its top position according other measures. Even in the rest of the years this fund could maintain its position and the rank. This example proves that the performance of the fund depends primarily on the selection of the measure. Thus a fund being the best according to one measure may not be, if not the worst, according another measure. The performance of many funds like that of Tata Pure Equity, UTI Dividend Yield, DSPBR Top 100 and Birla Frontline Equity, a few to quote, has been found to be very volatile over the period. A closer look into the ratios and the ranks reveals that there was no any fund that performed consistently during the years. This observation is contrasting with that of according to Sharpe, Treynor and Jensen measures. We also observe that the variance in the performance and ranking of the funds over five years is higher than that of Sharpe, Treynor and Jensen. This observation is also proved by hypothesis testing. As the observed 'p' value $(0.00)$ with respect to $\mathrm{H}_{5}$, is less than critical ' $p$ ' value $(0.05)$ we reject $\mathrm{HO}_{5}$ and conclude that the performance of sample funds varied over the years 2008-09 through 2012-13. This implies that the time factor played in the performance of the funds according to information ratio.

\subsection{Modigliani Squared ( $\left.\mathbf{M}^{2}\right)$}

$\mathrm{M}^{2}$ attempts to test the performance in terms of the possible diversification of non-systematic risk in the portfolio. For this purpose, the original portfolio will be adjusted to such possible diversification and therefore return of adjusted portfolio is taken into consideration. The adjusted portfolio return is then compared with the concerned benchmark portfolio return. Higher is the excess of adjusted portfolio return over the benchmark portfolio return better is the performance of the fund manager and vise versa. The increasing difference indicates the fund manager's ability to outperform the benchmark return. The $\mathrm{M}^{2}$ values of the sample funds over the years are mentioned in Table 10. Like in the case of Sharpe and Treynor measures, $\mathrm{M}^{2}$ values of all the funds in the years 2008-09 and 2011-12 were negative indicating that all the sample funds underperformed the benchmark portfolio concerned. The funds however, have outperformed the benchmark in the rest of the years. If we again focus our attention on ICICI Discovery fund, we observe that his fund performed better in many years. As we observe, its rank is 1, 4, 11, 7 and 2 during five years respectively. This is quite good when we compare with other funds. Of course the performance of the majority of the funds has been found to be inconsistent over the years. To quote a few, we find this kind of scenario in case of UTI Dividend Yield fund, Sundaram Tax Saver fund, SBI Contra fund, Principal Large Cap fund and a few more which could be traced out from Table 10. Barring a few cases as discussed above, the outperforming ability of the most of the fund managers varied over the period as it has been indicated by ranks. This is also proved through hypothesis testing. As the observed 'p' value $(0.00)$, with respect to $\mathrm{H}_{6}$, is less than the critical ' $p$ ' value $(0.05)$ we reject $\mathrm{H}_{6}$ and conclude that the performance of sample funds, according to $\mathrm{M}^{2}$, varied over the years 2008-09 through 2012-13. This again implies that the time factor played a role in the performance of sample funds according to $\mathrm{M}^{2}$.

\subsection{Net Selectivity}

The net selectivity indicates the fund manager's ability to justify the loss of diversification. This measure also indicates as to how much return comes from stock selection the credit of which goes to the fund manager. Higher the net selectivity greater is the stock selection ability of the manager and vise versa. The returns that are due to stock selection ability of sample fund managers are summarized in Table 11. Surprisingly the 
Table 7. Risk-adjusted performance and rank of sample funds according to Treynor Ratio (T.R)

\begin{tabular}{|c|c|c|c|c|c|c|c|c|c|c|}
\hline \multirow[b]{2}{*}{ Fund Name } & \multicolumn{2}{|c|}{$2008-09$} & \multicolumn{2}{|c|}{$2009-10$} & \multicolumn{2}{|c|}{$2010-11$} & \multicolumn{2}{|c|}{$2011-12$} & \multicolumn{2}{|c|}{$2012-13$} \\
\hline & T.R & Rank & T.R & Rank & T.R & Rank & T.R & Rank & T.R & Rank \\
\hline Birla Advantage & -0.675 & 42 & 0.775 & 45 & -0.018 & 37 & -0.215 & 46 & -0.031 & 28 \\
\hline Birla Equity & -0.755 & 47 & 0.924 & 41 & -0.028 & 40 & -0.177 & 43 & -0.010 & 19 \\
\hline Birla Frontline Equity & -0.537 & 27 & 0.937 & 39 & 0.046 & 22 & -0.185 & 44 & 0.053 & 2 \\
\hline Birla Midcap & -0.629 & 35 & 1.086 & 21 & -0.078 & 47 & -0.125 & 24 & -0.059 & 44 \\
\hline DSPBR Equity & -0.505 & 20 & 1.091 & 20 & 0.051 & 20 & -0.124 & 23 & -0.059 & 43 \\
\hline DSPBR Opp & -0.506 & 21 & 1.000 & 31 & 0.053 & 17 & -0.241 & 47 & -0.004 & 15 \\
\hline DSPBR Small \& Midcap & -0.511 & 22 & 1.379 & 7 & 0.057 & 15 & -0.114 & 16 & -0.054 & 40 \\
\hline DSPBR Tax Saver & -0.707 & 45 & 1.094 & 19 & 0.016 & 27 & -0.156 & 37 & 0.019 & 8 \\
\hline DSPBR Tiger & -0.490 & 17 & 0.807 & 44 & -0.053 & 43 & -0.147 & 35 & -0.057 & 42 \\
\hline DSPBR Top 100 & -0.410 & 7 & 0.994 & 32 & 0.051 & 21 & -0.108 & 11 & -0.046 & 37 \\
\hline Franklin India Bluechip & -0.380 & 5 & 0.972 & 36 & 0.065 & 12 & -0.119 & 21 & -0.021 & 26 \\
\hline Franklin India Prima & -0.617 & 33 & 1.231 & 12 & -0.014 & 34 & -0.082 & 4 & 0.034 & 5 \\
\hline Franklin India Taxshield & -0.475 & 15 & 1.068 & 22 & 0.078 & 8 & -0.110 & 14 & -0.011 & 21 \\
\hline HDFC Capital Builder & -0.496 & 19 & 1.205 & 13 & 0.077 & 9 & -0.151 & 36 & -0.018 & 24 \\
\hline HDFC Equity & -0.458 & 12 & 1.035 & 27 & 0.107 & 3 & -0.144 & 32 & -0.043 & 35 \\
\hline HDFC Growth & -0.544 & 29 & 0.976 & 35 & 0.115 & 2 & -0.104 & 9 & -0.041 & 34 \\
\hline HDFC Long Term Adv & -0.518 & 23 & 0.979 & 34 & 0.092 & 6 & -0.104 & 8 & -0.011 & 20 \\
\hline HDFC Tax Saver & -0.383 & 6 & 1.166 & 15 & 0.057 & 14 & -0.141 & 31 & -0.068 & 46 \\
\hline HDFC Top 200 & -0.364 & 4 & 0.953 & 37 & 0.095 & 5 & -0.141 & 30 & -0.034 & 30 \\
\hline ICICI Discovery & -0.242 & 2 & 1.447 & 4 & 0.066 & 11 & -0.099 & 7 & 0.065 & 1 \\
\hline ICICI Dynamic & -0.604 & 32 & 1.055 & 24 & 0.149 & 1 & -0.122 & 22 & -0.022 & 27 \\
\hline ICICI Midcap & -0.520 & 24 & 1.437 & 6 & -0.088 & 48 & -0.105 & 10 & -0.101 & 47 \\
\hline IDFC Classic Equity & -0.645 & 37 & 1.950 & 1 & -0.018 & 36 & -0.211 & 45 & 0.034 & 4 \\
\hline IDFC Premier Equity & -0.558 & 30 & 1.575 & 3 & 0.002 & 30 & -0.034 & 1 & 0.020 & 6 \\
\hline Principal Div Yield & -0.761 & 48 & 1.096 & 18 & 0.073 & 10 & -0.108 & 12 & -0.036 & 31 \\
\hline Principal Growth & -0.462 & 13 & 1.029 & 28 & -0.048 & 42 & -0.135 & 28 & 0.016 & 9 \\
\hline Principal Large Cap & -0.352 & 3 & 1.101 & 17 & 0.056 & 16 & -0.170 & 42 & -0.013 & 22 \\
\hline Principal Tax Savings & -0.680 & 43 & 1.051 & 25 & -0.042 & 41 & -0.129 & 27 & 0.020 & 7 \\
\hline Reliance Equity & -0.683 & 44 & 0.558 & 48 & -0.192 & 50 & -0.145 & 33 & 0.007 & 11 \\
\hline Reliance Equity Opp & -0.648 & 39 & 1.623 & 2 & 0.063 & 13 & -0.056 & 3 & 0.037 & 3 \\
\hline Reliance Growth & -0.633 & 36 & 1.067 & 23 & -0.015 & 35 & -0.125 & 25 & -0.038 & 32 \\
\hline Reliance Regular Savings & -0.490 & 18 & 1.039 & 26 & -0.003 & 33 & -0.145 & 34 & -0.005 & 16 \\
\hline Reliance Tax Saver & -0.538 & 28 & 1.148 & 16 & 0.051 & 19 & -0.055 & 2 & -0.044 & 36 \\
\hline Reliance Vision Fund & -0.621 & 34 & 0.986 & 33 & 0.009 & 28 & -0.109 & 13 & -0.111 & 48 \\
\hline SBI Contra & -0.455 & 11 & 0.927 & 40 & -0.063 & 45 & -0.140 & 29 & 0.014 & 10 \\
\hline SBI Magnum Equity & -0.536 & 26 & 0.765 & 46 & 0.053 & 18 & -0.116 & 17 & 0.006 & 12 \\
\hline SBI Magnum Tax Gain & -0.485 & 16 & 0.952 & 38 & -0.025 & 39 & -0.117 & 20 & -0.008 & 17 \\
\hline Sundaram Equity Mulitplier & -1.025 & 49 & 0.860 & 43 & -0.024 & 38 & -0.159 & 39 & -0.040 & 33 \\
\hline Sundaram Growth Fund & -0.658 & 40 & 0.887 & 42 & 0.018 & 26 & -0.160 & 40 & -0.048 & 38 \\
\hline Sundaram Select Focus & -1.228 & 50 & 0.714 & 47 & -0.002 & 32 & -0.331 & 49 & -0.017 & 23 \\
\hline Sundaram Select Midcap & -0.667 & 41 & 1.361 & 8 & 0.023 & 25 & -0.111 & 15 & -0.021 & 25 \\
\hline Sundaram Smile & -0.446 & 10 & 1.023 & 30 & -0.062 & 44 & -0.158 & 38 & -0.264 & 49 \\
\hline Sundaram Tax Saver & 0.846 & 1 & -17.693 & 50 & -0.138 & 49 & -2.153 & 50 & -14.541 & 50 \\
\hline Tata Equity 0pp & -0.593 & 31 & 1.329 & 10 & -0.069 & 46 & -0.093 & 6 & -0.009 & 18 \\
\hline Tata PE Fund & -0.647 & 38 & 1.203 & 14 & 0.027 & 24 & -0.087 & 5 & -0.055 & 41 \\
\hline Tata Pure Equity & -0.713 & 46 & 1.024 & 29 & -0.002 & 31 & -0.117 & 18 & 0.002 & 13 \\
\hline Tata Tax Saving & -0.525 & 25 & 0.514 & 49 & 0.042 & 23 & -0.273 & 48 & -0.031 & 29 \\
\hline UTI Dividend Yield & -0.415 & 8 & 1.444 & 5 & 0.097 & 4 & -0.163 & 41 & -0.064 & 45 \\
\hline UTI Equity & -0.435 & 9 & 1.254 & 11 & 0.087 & 7 & -0.117 & 19 & -0.001 & 14 \\
\hline UTI Midcap & -0.464 & 14 & 1.358 & 9 & 0.008 & 29 & -0.126 & 26 & -0.052 & 39 \\
\hline
\end{tabular}


Table 8. Risk-adjusted performance and rank of sample funds according to Jensen's Alpha (J.A)

\begin{tabular}{|c|c|c|c|c|c|c|c|c|c|c|}
\hline \multirow[b]{2}{*}{ Fund Name } & \multicolumn{2}{|c|}{ 2008-09 } & \multicolumn{2}{|c|}{$2009-10$} & \multicolumn{2}{|c|}{$2010-11$} & \multicolumn{2}{|c|}{ 2011-12 } & \multicolumn{2}{|c|}{$2012-13$} \\
\hline & J.A & Rank & J.A & Rank & J.A & Rank & J.A & Rank & J.A & Rank \\
\hline Birla Advantage & -13.81 & 41 & -13.78 & 47 & -4.32 & 36 & -3.31 & 46 & -1.36 & 35 \\
\hline Birla Equity & -17.59 & 46 & 4.06 & 41 & -5.96 & 41 & -0.08 & 41 & 1.61 & 20 \\
\hline Birla Frontline Equity & -3.90 & 23 & 5.06 & 40 & 2.68 & 20 & -0.75 & 43 & 8.12 & 6 \\
\hline Birla Midcap & -8.56 & 30 & -18.02 & 48 & -5.54 & 40 & -0.01 & 40 & 5.33 & 14 \\
\hline DSPBR Equity & -2.09 & 21 & 20.55 & 22 & 4.80 & 17 & 4.77 & 21 & -3.94 & 41 \\
\hline DSPBR Opp & -2.52 & 22 & 13.61 & 29 & 5.60 & 14 & -5.16 & 47 & 3.33 & 15 \\
\hline DSPBR Small \& Midcap & 1.74 & 13 & 15.76 & 28 & 8.37 & 3 & 0.96 & 37 & 8.12 & 5 \\
\hline DSPBR Tax Saver & -14.59 & 43 & 22.27 & 17 & 0.71 & 24 & 1.41 & 35 & 6.00 & 11 \\
\hline DSPBR Tiger & -1.35 & 18 & -3.03 & 45 & -9.28 & 48 & 3.55 & 28 & -7.65 & 48 \\
\hline DSPBR Top 100 & 5.32 & 8 & 11.12 & 33 & 2.59 & 21 & 6.60 & 11 & -3.92 & 39 \\
\hline Franklin India Bluechip & 7.58 & 6 & 18.57 & 27 & 1.81 & 23 & 6.49 & 12 & -2.57 & 37 \\
\hline Franklin India Prima & -12.22 & 38 & 40.84 & 5 & -3.25 & 32 & 8.29 & 9 & 9.52 & 3 \\
\hline Franklin India Taxshield & 0.19 & 16 & 18.81 & 26 & 6.66 & 10 & 4.44 & 22 & 1.79 & 18 \\
\hline HDFC Capital Builder & -1.77 & 20 & 31.85 & 9 & 7.54 & 5 & 1.81 & 33 & 0.92 & 25 \\
\hline HDFC Equity & 1.73 & 14 & 21.58 & 18 & 12.34 & 1 & 3.05 & 29 & -1.32 & 33 \\
\hline HDFC Growth & -7.30 & 29 & 19.21 & 25 & 6.99 & 8 & 9.13 & 6 & -5.66 & 46 \\
\hline HDFC Long Term Adv & -5.48 & 27 & 21.40 & 19 & 5.15 & 16 & 9.94 & 5 & -1.12 & 30 \\
\hline HDFC Tax Saver & 10.55 & 4 & 30.38 & 10 & 5.74 & 12 & 2.72 & 30 & -3.93 & 40 \\
\hline HDFC Top 200 & 12.19 & 3 & 6.62 & 39 & 8.40 & 2 & 3.71 & 25 & -1.35 & 34 \\
\hline ICICI Discovery & 52.25 & 1 & 24.43 & 13 & 6.84 & 9 & 2.18 & 31 & 12.47 & 2 \\
\hline ICICI Dynamic & -10.91 & 34 & 30.04 & 11 & 5.67 & 13 & 5.79 & 15 & -1.20 & 31 \\
\hline ICICI Midcap & 1.19 & 15 & 20.02 & 23 & -7.44 & 45 & 2.02 & 32 & 1.54 & 21 \\
\hline IDFC Classic Equity & -11.17 & 36 & 73.07 & 2 & -4.06 & 34 & -2.93 & 44 & 7.22 & 7 \\
\hline IDFC Premier Equity & -4.24 & 25 & 50.92 & 4 & -1.63 & 29 & 10.34 & 4 & 7.13 & 8 \\
\hline Principal Div Yield & -16.32 & 45 & 19.79 & 24 & 7.39 & 7 & 7.31 & 10 & -0.71 & 29 \\
\hline Principal Growth & 2.70 & 11 & 10.22 & 34 & -6.71 & 44 & 4.85 & 20 & 5.40 & 13 \\
\hline Principal Large Cap & 14.89 & 2 & 22.92 & 16 & 3.07 & 19 & 0.56 & 39 & 0.01 & 28 \\
\hline Principal Tax Savings & -17.66 & 47 & 11.74 & 32 & -6.56 & 43 & 5.39 & 18 & 5.97 & 12 \\
\hline Reliance Equity & -13.75 & 40 & -13.65 & 46 & -14.15 & 50 & 3.95 & 24 & 2.61 & 17 \\
\hline Reliance Equity 0pp & -11.96 & 37 & 60.67 & 3 & 5.45 & 15 & 13.85 & 2 & 6.68 & 10 \\
\hline Reliance Growth & -11.14 & 35 & 22.98 & 15 & -4.08 & 35 & 5.72 & 16 & -4.38 & 43 \\
\hline Reliance Regular Savings & -1.37 & 19 & 20.87 & 21 & -3.07 & 31 & 3.61 & 27 & 1.65 & 19 \\
\hline Reliance Tax Saver & -4.40 & 26 & 23.77 & 14 & 3.64 & 18 & 16.53 & 1 & -5.31 & 45 \\
\hline Reliance Vision Fund & -9.87 & 33 & 12.50 & 31 & -1.54 & 28 & 8.87 & 7 & -12.96 & 50 \\
\hline SBI Contra & 1.88 & 12 & 7.93 & 38 & -8.81 & 47 & 3.96 & 23 & 2.85 & 16 \\
\hline SBI Magnum Equity & -8.62 & 31 & 8.20 & 37 & 0.28 & 25 & 5.92 & 14 & 1.26 & 23 \\
\hline SBI Magnum Tax Gain & -0.92 & 17 & 9.83 & 36 & -4.46 & 37 & 5.67 & 17 & 0.67 & 26 \\
\hline Sundaram Equity Mulitplier & -24.82 & 48 & 2.09 & 42 & -3.39 & 33 & 1.08 & 36 & -1.65 & 36 \\
\hline Sundaram Growth Fund & -14.20 & 42 & 0.06 & 44 & -0.17 & 26 & 1.55 & 34 & -4.16 & 42 \\
\hline Sundaram Select Focus & -30.36 & 49 & 1.93 & 43 & -6.40 & 42 & -9.18 & 49 & -1.24 & 32 \\
\hline Sundaram Select Midcap & -4.24 & 24 & 9.95 & 35 & 7.66 & 4 & 3.66 & 26 & 9.25 & 4 \\
\hline Sundaram Smile & 9.22 & 5 & -24.44 & 49 & -5.17 & 38 & -2.97 & 45 & -6.07 & 47 \\
\hline Sundaram Tax Saver & -52.92 & 50 & 89.72 & 1 & -11.11 & 49 & -15.71 & 50 & 14.00 & 1 \\
\hline Tata Equity 0pp & -9.66 & 32 & 34.08 & 8 & -8.27 & 46 & 8.42 & 8 & 1.16 & 24 \\
\hline Tata PE Fund & -12.81 & 39 & 38.15 & 6 & -1.72 & 30 & 12.67 & 3 & -10.18 & 49 \\
\hline Tata Pure Equity & -14.59 & 44 & 21.00 & 20 & -5.52 & 39 & 6.39 & 13 & 0.19 & 27 \\
\hline Tata Tax Saving & -6.14 & 28 & -24.70 & 50 & -0.43 & 27 & -5.91 & 48 & -3.83 & 38 \\
\hline UTI Dividend Yield & 4.30 & 9 & 34.67 & 7 & 7.46 & 6 & 0.91 & 38 & -5.18 & 44 \\
\hline UTI Equity & 3.48 & 10 & 25.93 & 12 & 6.34 & 11 & 5.09 & 19 & 1.32 & 22 \\
\hline UTI Midcap & 7.13 & 7 & 12.83 & 30 & 2.57 & 22 & -0.14 & 42 & 6.98 & 9 \\
\hline
\end{tabular}


Table 9. Risk-adjusted performance and rank of sample funds according to Information Ratio

\begin{tabular}{|c|c|c|c|c|c|c|c|c|c|c|}
\hline \multirow[b]{2}{*}{ Fund } & \multicolumn{2}{|c|}{ 2008-09 } & \multicolumn{2}{|c|}{ 2009-10 } & \multicolumn{2}{|c|}{ 2010-11 } & \multicolumn{2}{|c|}{ 2011-12 } & \multicolumn{2}{|c|}{$2012-13$} \\
\hline & I.R & Rank & I.R & Rank & I.R & Rank & I.R & Rank & I.R & Rank \\
\hline Birla Advantage & -0.25 & 39 & 1.72 & 29 & -3.95 & 41 & -0.50 & 47 & -2.51 & 37 \\
\hline Birla Equity & -0.35 & 40 & 2.79 & 24 & -24.18 & 48 & 0.22 & 41 & 2.23 & 16 \\
\hline Birla Frontline Equity & 4.11 & 8 & 1.70 & 30 & 3.78 & 9 & 2.75 & 22 & 16.96 & 1 \\
\hline Birla Midcap & -0.99 & 43 & 7.11 & 15 & -2.05 & 35 & 2.88 & 20 & 3.74 & 9 \\
\hline DSPBR Equity & 5.32 & 6 & 1.59 & 31 & 2.83 & 14 & 2.19 & 26 & -5.70 & 45 \\
\hline DSPBR Opp & 6.61 & 5 & 0.02 & 38 & 2.63 & 16 & -0.45 & 46 & 1.80 & 20 \\
\hline DSPBR Small \& Midcap & 1.87 & 18 & 8.72 & 13 & 5.97 & 4 & 0.78 & 39 & 2.69 & 15 \\
\hline DSPBR Tax Saver & 1.02 & 26 & 7.34 & 14 & 0.46 & 25 & 3.46 & 13 & 4.51 & 5 \\
\hline DSPBR Tiger & 0.43 & 35 & -4.15 & 46 & -2.57 & 38 & -0.14 & 44 & -4.15 & 42 \\
\hline DSPBR Top 100 & 41.33 & 1 & -4.51 & 47 & 3.61 & 11 & 9.30 & 2 & -2.83 & 39 \\
\hline Franklin India Bluechip & 2.45 & 16 & 3.31 & 21 & 2.41 & 18 & 4.24 & 8 & -1.43 & 34 \\
\hline Franklin India Prima & -2.23 & 48 & 37.13 & 3 & -2.45 & 37 & 5.04 & 5 & 4.45 & 6 \\
\hline Franklin India Taxshield & 3.06 & 13 & 1.30 & 33 & 3.64 & 10 & 8.08 & 3 & 3.32 & 12 \\
\hline HDFC Capital Builder & 0.76 & 33 & 11.23 & 9 & 5.32 & 7 & 3.24 & 16 & 1.09 & 24 \\
\hline HDFC Equity & 1.64 & 22 & 51.51 & 2 & 7.83 & 1 & 1.34 & 32 & -2.12 & 35 \\
\hline HDFC Growth & 0.14 & 37 & 9.39 & 11 & 1.58 & 21 & 3.39 & 14 & -11.39 & 49 \\
\hline HDFC Long Term Adv & 0.02 & 38 & 13.92 & 7 & 2.13 & 19 & 2.71 & 23 & -1.01 & 32 \\
\hline HDFC Tax Saver & 0.89 & 30 & 11.47 & 8 & 3.56 & 12 & 2.77 & 21 & -4.62 & 43 \\
\hline HDFC Top 200 & 3.92 & 10 & 68.34 & 1 & 6.50 & 3 & 4.72 & 6 & -2.28 & 36 \\
\hline ICICI Discovery & 0.87 & 31 & 6.60 & 17 & 5.73 & 5 & 1.61 & 29 & 14.72 & 2 \\
\hline ICICI Dynamic & 2.45 & 15 & 8.84 & 12 & 2.56 & 17 & 3.96 & 11 & -0.73 & 30 \\
\hline ICICI Midcap & -2.57 & 49 & 6.90 & 16 & -8.83 & 46 & 0.90 & 37 & 3.38 & 11 \\
\hline IDFC Classic Equity & 1.74 & 20 & 1.76 & 28 & -26.46 & 49 & -0.61 & 49 & 6.06 & 3 \\
\hline IDFC Premier Equity & 2.98 & 14 & 19.22 & 4 & -1.13 & 30 & 2.98 & 18 & 4.03 & 7 \\
\hline Principal Div Yield & 0.94 & 28 & 0.03 & 37 & 3.24 & 13 & 2.22 & 25 & -0.89 & 31 \\
\hline Principal Growth & -1.27 & 44 & -5.93 & 49 & -3.07 & 39 & 1.58 & 30 & 2.96 & 13 \\
\hline Principal Large Cap & 1.69 & 21 & 4.17 & 20 & 1.15 & 24 & 1.30 & 34 & -0.23 & 29 \\
\hline Principal Tax Savings & -5.30 & 50 & -5.86 & 48 & -3.31 & 40 & 2.12 & 28 & 3.61 & 10 \\
\hline Reliance Equity & 1.62 & 23 & -12.99 & 50 & -7.22 & 45 & -0.37 & 45 & 2.05 & 18 \\
\hline Reliance Equity Opp & 3.18 & 11 & 9.88 & 10 & 2.82 & 15 & 3.77 & 12 & 3.91 & 8 \\
\hline Reliance Growth & 0.95 & 27 & 16.88 & 6 & -1.55 & 34 & 1.33 & 33 & -3.01 & 40 \\
\hline Reliance Regular Savings & 1.55 & 24 & 16.99 & 5 & -1.51 & 33 & 0.26 & 40 & 0.20 & 27 \\
\hline Reliance Tax Saver & 4.02 & 9 & 2.12 & 27 & 1.16 & 23 & 2.35 & 24 & -2.82 & 38 \\
\hline Reliance Vision Fund & 1.77 & 19 & 0.10 & 36 & -0.46 & 27 & 1.12 & 35 & -6.80 & 47 \\
\hline SBI Contra & 3.14 & 12 & -0.17 & 39 & -4.53 & 42 & 1.38 & 31 & 1.67 & 21 \\
\hline SBI Magnum Equity & -1.64 & 45 & 5.24 & 18 & -0.18 & 26 & 5.64 & 4 & 1.26 & 23 \\
\hline SBI Magnum Tax Gain & 0.60 & 34 & -0.60 & 42 & -2.35 & 36 & 4.17 & 9 & 0.45 & 25 \\
\hline Sundaram Equity Mulitplier & 0.36 & 36 & -1.29 & 44 & -5.10 & 44 & 0.95 & 36 & -3.18 & 41 \\
\hline Sundaram Growth Fund & -0.96 & 42 & 0.26 & 35 & 1.19 & 22 & 2.14 & 27 & -7.31 & 48 \\
\hline Sundaram Select Focus & -1.68 & 46 & 2.18 & 26 & -5.03 & 43 & -2.00 & 50 & -1.23 & 33 \\
\hline Sundaram Select Midcap & 4.11 & 7 & 2.83 & 23 & 7.21 & 2 & 3.12 & 17 & 1.99 & 19 \\
\hline Sundaram Smile & 2.06 & 17 & 1.47 & 32 & -12.91 & 47 & -0.57 & 48 & 0.09 & 28 \\
\hline Sundaram Tax Saver & 1.03 & 25 & -0.20 & 40 & -1.26 & 31 & 0.10 & 42 & 6.01 & 4 \\
\hline Tata Equity 0pp & -1.68 & 47 & 2.77 & 25 & -26.65 & 50 & 4.12 & 10 & 2.22 & 17 \\
\hline Tata PE Fund & 0.79 & 32 & 5.03 & 19 & -0.94 & 29 & 3.37 & 15 & -6.15 & 46 \\
\hline Tata Pure Equity & 16.97 & 4 & 3.03 & 22 & -1.48 & 32 & 4.30 & 7 & 0.24 & 26 \\
\hline Tata Tax Saving & -0.64 & 41 & -3.55 & 45 & -0.72 & 28 & -0.07 & 43 & -5.38 & 44 \\
\hline UTI Dividend Yield & 25.79 & 2 & -0.26 & 41 & 5.68 & 6 & 2.93 & 19 & -12.83 & 50 \\
\hline UTI Equity & 21.96 & 3 & -0.84 & 43 & 4.77 & 8 & 11.51 & 1 & 1.44 & 22 \\
\hline UTI Midcap & 0.90 & 29 & 0.73 & 34 & 2.08 & 20 & 0.81 & 38 & 2.71 & 14 \\
\hline
\end{tabular}


Table 10. Risk-adjusted performance and rank of sample funds according to $\mathrm{M}^{2}$

\begin{tabular}{|c|c|c|c|c|c|c|c|c|c|c|}
\hline \multirow[b]{2}{*}{ Fund } & \multicolumn{2}{|c|}{ 2008-09 } & \multicolumn{2}{|c|}{$2009-10$} & \multicolumn{2}{|c|}{$2010-11$} & \multicolumn{2}{|c|}{$2011-12$} & \multicolumn{2}{|c|}{$2012-13$} \\
\hline & $M^{2}$ & Rank & $M^{2}$ & Rank & $M^{2}$ & Rank & $M^{2}$ & Rank & $M^{2}$ & Rank \\
\hline Birla Advantage & -0.58 & 44 & 0.81 & 46 & 0.05 & 36 & -0.13 & 46 & 0.05 & 29 \\
\hline Birla Equity & -0.66 & 48 & 0.96 & 42 & 0.03 & 40 & -0.09 & 43 & 0.07 & 20 \\
\hline Birla Frontline Equity & -0.45 & 28 & 0.97 & 40 & 0.11 & 22 & -0.10 & 44 & 0.14 & 3 \\
\hline Birla Midcap & -0.55 & 39 & 1.12 & 20 & -0.01 & 48 & -0.04 & 26 & 0.02 & 44 \\
\hline DSPBR Equity & -0.42 & 23 & 1.12 & 21 & 0.11 & 19 & -0.04 & 22 & 0.02 & 45 \\
\hline DSPBR Opp & -0.43 & 25 & 1.04 & 32 & 0.11 & 17 & -0.15 & 47 & 0.08 & 17 \\
\hline DSPBR Small \& Midcap & -0.42 & 22 & 1.42 & 6 & 0.12 & 15 & -0.03 & 16 & 0.03 & 41 \\
\hline DSPBR Tax Saver & -0.59 & 46 & 1.13 & 18 & 0.08 & 27 & -0.07 & 39 & 0.10 & 9 \\
\hline DSPBR Tiger & -0.40 & 16 & 0.85 & 45 & 0.01 & 43 & -0.06 & 32 & 0.03 & 43 \\
\hline DSPBR Top 100 & -0.34 & 7 & 1.02 & 33 & 0.11 & 18 & -0.02 & 13 & 0.04 & 38 \\
\hline Franklin India Bluechip & -0.30 & 6 & 1.00 & 37 & 0.13 & 12 & -0.03 & 21 & 0.06 & 28 \\
\hline Franklin India Prima & -0.52 & 36 & 1.27 & 11 & 0.05 & 34 & 0.00 & 4 & 0.12 & 6 \\
\hline Franklin India Taxshield & -0.39 & 15 & 1.10 & 24 & 0.14 & 8 & -0.03 & 15 & 0.07 & 21 \\
\hline HDFC Capital Builder & -0.41 & 20 & 1.24 & 12 & 0.14 & 9 & -0.07 & 36 & 0.07 & 24 \\
\hline HDFC Equity & -0.36 & 9 & 1.08 & 28 & 0.17 & 2 & -0.06 & 34 & 0.04 & 37 \\
\hline HDFC Growth & -0.40 & 17 & 1.02 & 36 & 0.16 & 3 & -0.02 & 8 & 0.04 & 35 \\
\hline HDFC Long Term Adv & -0.43 & 24 & 1.02 & 34 & 0.15 & 6 & -0.02 & 9 & 0.07 & 22 \\
\hline HDFC Tax Saver & -0.29 & 5 & 1.20 & 13 & 0.12 & 14 & -0.06 & 30 & 0.01 & 47 \\
\hline HDFC Top 200 & -0.28 & 4 & 1.00 & 38 & 0.16 & 5 & -0.06 & 31 & 0.05 & 31 \\
\hline ICICI Discovery & -0.12 & 1 & 1.46 & 4 & 0.13 & 11 & -0.01 & 7 & 0.15 & 2 \\
\hline ICICI Dynamic & -0.51 & 34 & 1.09 & 25 & 0.21 & 1 & -0.04 & 23 & 0.06 & 27 \\
\hline ICICI Midcap & -0.41 & 18 & 1.48 & 3 & -0.03 & 49 & -0.02 & 10 & -0.02 & 48 \\
\hline IDFC Classic Equity & -0.55 & 40 & 1.12 & 22 & 0.04 & 37 & -0.13 & 45 & 0.12 & 5 \\
\hline IDFC Premier Equity & -0.46 & 30 & 1.61 & 2 & 0.06 & 30 & 0.05 & 1 & 0.10 & 8 \\
\hline Principal Div Yield & -0.57 & 42 & 1.13 & 19 & 0.13 & 10 & -0.02 & 12 & 0.05 & 32 \\
\hline Principal Growth & -0.37 & 13 & 1.06 & 29 & 0.02 & 42 & -0.05 & 28 & 0.10 & 10 \\
\hline Principal Large Cap & -0.27 & 3 & 1.13 & 17 & 0.12 & 16 & -0.09 & 42 & 0.07 & 23 \\
\hline Principal Tax Savings & -0.59 & 45 & 1.08 & 26 & 0.02 & 41 & -0.05 & 27 & 0.10 & 7 \\
\hline Reliance Equity & -0.53 & 37 & 0.60 & 49 & -0.11 & 50 & -0.06 & 35 & 0.09 & 12 \\
\hline Reliance Equity 0pp & -0.57 & 43 & 1.63 & 1 & 0.12 & 13 & 0.03 & 3 & 0.12 & 4 \\
\hline Reliance Growth & -0.52 & 35 & 1.11 & 23 & 0.05 & 35 & -0.04 & 24 & 0.04 & 33 \\
\hline Reliance Regular Savings & -0.42 & 21 & 1.08 & 27 & 0.06 & 33 & -0.06 & 33 & 0.08 & 16 \\
\hline Reliance Tax Saver & -0.44 & 26 & 1.18 & 16 & 0.11 & 21 & 0.03 & 2 & 0.04 & 36 \\
\hline Reliance Vision Fund & -0.50 & 33 & 1.02 & 35 & 0.07 & 28 & -0.02 & 11 & -0.03 & 50 \\
\hline SBI Contra & -0.38 & 14 & 0.97 & 41 & 0.00 & 44 & -0.06 & 29 & 0.10 & 11 \\
\hline SBI Magnum Equity & -0.46 & 29 & 0.80 & 47 & 0.11 & 20 & -0.03 & 18 & 0.09 & 13 \\
\hline SBI Magnum Tax Gain & -0.41 & 19 & 0.99 & 39 & 0.04 & 38 & -0.03 & 19 & 0.07 & 18 \\
\hline Sundaram Equity Mulitplier & -0.77 & 49 & 0.89 & 44 & 0.04 & 39 & -0.07 & 37 & 0.04 & 34 \\
\hline Sundaram Growth Fund & -0.48 & 31 & 0.92 & 43 & 0.08 & 26 & -0.08 & 41 & 0.03 & 39 \\
\hline Sundaram Select Focus & -1.04 & 50 & 0.75 & 48 & 0.06 & 32 & -0.24 & 49 & 0.07 & 25 \\
\hline Sundaram Select Midcap & -0.57 & 41 & 1.40 & 7 & 0.09 & 25 & -0.03 & 14 & 0.06 & 26 \\
\hline Sundaram Smile & -0.36 & 11 & 1.06 & 31 & 0.00 & 45 & -0.07 & 38 & -0.02 & 49 \\
\hline Sundaram Tax Saver & -0.17 & 2 & 1.34 & 9 & -0.01 & 47 & -0.33 & 50 & 0.40 & 1 \\
\hline Tata Equity 0pp & -0.50 & 32 & 1.33 & 10 & -0.01 & 46 & -0.01 & 6 & 0.07 & 19 \\
\hline Tata PE Fund & -0.54 & 38 & 1.20 & 14 & 0.09 & 24 & 0.00 & 5 & 0.03 & 42 \\
\hline Tata Pure Equity & -0.64 & 47 & 1.06 & 30 & 0.06 & 31 & -0.03 & 17 & 0.08 & 14 \\
\hline Tata Tax Saving & -0.45 & 27 & 0.53 & 50 & 0.10 & 23 & -0.16 & 48 & 0.05 & 30 \\
\hline UTI Dividend Yield & -0.34 & 8 & 1.42 & 5 & 0.16 & 4 & -0.08 & 40 & 0.02 & 46 \\
\hline UTI Equity & -0.36 & 10 & 1.19 & 15 & 0.15 & 7 & -0.03 & 20 & 0.08 & 15 \\
\hline UTI Midcap & -0.37 & 12 & 1.37 & 8 & 0.07 & 29 & -0.04 & 25 & 0.03 & 40 \\
\hline
\end{tabular}


performance of the most of the sample funds is very good in the years 2008-09 and 2011-12 unlike in case of Sharpe and Treynor measures. The data reveals that almost all sample fund managers exhibited their stock selection ability and maximized the portfolio return during the entire period of study. There were however a few funds managers (like that of Birla Midcap, Birla Equity, Principal Tax Savings, reliance Equity and a few others) whose stock selectivity ability had negative impact on portfolio return during a couple of years. However we observe extremely varying stock selection ability of the fund managers over the years. For instance, in case of Sundaram Tax Saver fund, the selection ability was the best in 2008-09 and 2012-13 and was worst in 2010-11 and 2011-12. Also in case of UTI Midcap, it was good in the first and the last years and otherwise during three years in between. Again the most talked about fund in our study, ICICI Discovery fund exhibited very good stock selection ability almost during all the years. Barring the first year's ability, Reliance Equity Opportunity funds also falls in this category. We have been talking about these funds throughout our discussions for their superior performance among the sample funds. On an aggregate basis, however the stock selection ability of the fund managers varied over the years and we observe that majority of the fund managers failed to maintain the consistency in this regard. The variance in the performance of funds over the years is tested through $\mathrm{HO}_{7}$. As the observed 'p' value $(0.00)$ is less than the critical ' $p$ ' value $(0.05)$ we reject $\mathrm{H}_{7}$ and conclude that the performance of the sample funds according to net selectivity varied over the years 2008-09 through 2012-13. This implies that time factor played a role in the performance of sample funds even according net selectivity.

\subsection{Overall Composite Ranking}

The ranks of all the funds according to all selected performance measures over five years have been captured in Table 12. Though these ranks are not different from the ones available in earlier tables concerned, Table 12 provides a consolidated picture of fund-wise and measure-wise rankings for each and every year. This facilitates us to scout if there were any funds that maintained consistent ranking according to all measures in a year or more. As we have discussed a lot about, ICICI Discovery fund again has been ranked as one of top performing funds according to all measures almost during the entire period of study. However Jensen's alpha and information ratio of this fund reveal that it did not perform better in the years 2009-10 through 2011-12. Two funds HDFC Top 200 and DSPBR Top 100 have been ranked among top 10 in the year 2008-09. Similarly some funds like Franklin India Prima, Birla Frontline Equity, HDFC Capital Builder, HDFC Equity, IDFC Premier Equity and few more performed equally well according to all measures in a particular year or the other. On an aggregate basis, there is no fund that performed either equally well or equally bad according all measures during the study period. Thus the observation we make is that selection of the measure plays a role in evaluating and analyzing the performance of a given fund. We understand this observation through a deeper look into Table 12.

\section{Conclusions and Scope for Further Study}

Portfolio performance evaluation is very critical while choosing a fund for investment and we understand that there is no any best method available to evaluate the performance. Apart from a measure, a host of other factors also influence the performance. Some of such factors are time period and market conditions. The performance thus gets influenced by the market factors and the factors specific to the fund. The variability caused by these factors is classified into systematic and non-systematic risks. Thus our study reveals that performance of a fund primarily depends on the selection of the measure. The study also reveals that frequency of measurement and the time factor play a crucial role in the performance analysis of the funds. Despite very interesting findings, the study suffers from a few limitations such as sample selection and limited literature review. However the study provides a basis for proving a few hypotheses with empirical analysis and thereby contributes its own to the body of knowledge. The study may be continued further in any possible direction and hence we sincerely invite the prospective researchers to take ahead. 
Table 11. Risk-adjusted performance and rank of sample funds according to Net Selectivity

\begin{tabular}{|c|c|c|c|c|c|c|c|c|c|c|}
\hline \multirow[b]{2}{*}{ Fund } & \multicolumn{2}{|c|}{$2008-09$} & \multicolumn{2}{|c|}{$2009-10$} & \multicolumn{2}{|c|}{$2010-11$} & \multicolumn{2}{|c|}{ 2011-12 } & \multicolumn{2}{|c|}{ 2012-13 } \\
\hline & N.S & Rank & N.S & Rank & N.S & Rank & N.S & Rank & N.S & Rank \\
\hline Birla Advantage & -0.05 & 44 & -0.11 & 47 & 0.00 & 37 & 0.05 & 46 & 0.07 & 35 \\
\hline Birla Equity & -0.09 & 47 & 0.07 & 41 & -0.02 & 41 & 0.08 & 41 & 0.10 & 21 \\
\hline Birla Frontline Equity & 0.04 & 27 & 0.09 & 40 & 0.07 & 20 & 0.08 & 43 & 0.16 & 5 \\
\hline Birla Midcap & -0.01 & 33 & -0.15 & 48 & -0.01 & 38 & 0.08 & 42 & 0.14 & 12 \\
\hline DSPBR Equity & 0.06 & 22 & 0.24 & 23 & 0.09 & 16 & 0.13 & 20 & 0.04 & 42 \\
\hline DSPBR Opp & 0.05 & 25 & 0.17 & 30 & 0.09 & 13 & 0.03 & 48 & 0.12 & 14 \\
\hline DSPBR Small \& Midcap & 0.11 & 14 & 0.20 & 29 & 0.12 & 3 & 0.10 & 38 & 0.17 & 4 \\
\hline DSPBR Tax Saver & -0.05 & 45 & 0.26 & 16 & 0.05 & 24 & 0.10 & 36 & 0.14 & 11 \\
\hline DSPBR Tiger & 0.08 & 18 & 0.03 & 45 & -0.06 & 48 & 0.13 & 24 & 0.01 & 48 \\
\hline DSPBR Top 100 & 0.13 & 10 & 0.15 & 33 & 0.07 & 18 & 0.15 & 13 & 0.04 & 39 \\
\hline Franklin India Bluechip & 0.16 & 8 & 0.22 & 28 & 0.06 & 22 & 0.15 & 11 & 0.06 & 38 \\
\hline Franklin India Prima & -0.04 & 39 & 0.45 & 4 & 0.00 & 33 & 0.17 & 9 & 0.18 & 3 \\
\hline Franklin India Taxshield & 0.09 & 17 & 0.22 & 27 & 0.11 & 8 & 0.13 & 23 & 0.10 & 18 \\
\hline HDFC Capital Builder & 0.07 & 20 & 0.36 & 8 & 0.12 & 5 & 0.10 & 33 & 0.09 & 26 \\
\hline HDFC Equity & 0.12 & 12 & 0.26 & 18 & 0.16 & 1 & 0.11 & 29 & 0.07 & 33 \\
\hline HDFC Growth & 0.06 & 23 & 0.23 & 26 & 0.10 & 11 & 0.18 & 6 & 0.03 & 47 \\
\hline HDFC Long Term Adv & 0.03 & 28 & 0.26 & 19 & 0.09 & 15 & 0.19 & 5 & 0.07 & 32 \\
\hline HDFC Tax Saver & 0.22 & 4 & 0.34 & 9 & 0.10 & 12 & 0.11 & 30 & 0.04 & 40 \\
\hline HDFC Top 200 & 0.21 & 5 & 0.11 & 39 & 0.12 & 2 & 0.12 & 28 & 0.07 & 36 \\
\hline ICICI Discovery & 0.83 & 1 & 0.26 & 17 & 0.11 & 6 & 0.11 & 31 & 0.21 & 2 \\
\hline ICICI Dynamic & -0.03 & 36 & 0.34 & 10 & 0.11 & 9 & 0.14 & 17 & 0.07 & 31 \\
\hline ICICI Midcap & 0.14 & 9 & 0.24 & 24 & -0.03 & 45 & 0.11 & 32 & 0.10 & 20 \\
\hline IDFC Classic Equity & -0.03 & 37 & 0.28 & 12 & 0.00 & 34 & 0.06 & 45 & 0.15 & 7 \\
\hline IDFC Premier Equity & 0.05 & 24 & 0.55 & 2 & 0.02 & 30 & 0.20 & 4 & 0.15 & 8 \\
\hline Principal Div Yield & -0.04 & 42 & 0.24 & 25 & 0.11 & 7 & 0.16 & 10 & 0.08 & 30 \\
\hline Principal Growth & 0.12 & 11 & 0.14 & 35 & -0.03 & 42 & 0.13 & 21 & 0.14 & 13 \\
\hline Principal Large Cap & 0.24 & 3 & 0.26 & 15 & 0.07 & 17 & 0.09 & 39 & 0.08 & 29 \\
\hline Principal Tax Savings & -0.09 & 48 & 0.15 & 32 & -0.03 & 43 & 0.14 & 18 & 0.14 & 10 \\
\hline Reliance Equity & -0.03 & 38 & -0.10 & 46 & -0.10 & 50 & 0.13 & 25 & 0.11 & 16 \\
\hline Reliance Equity Opp & -0.05 & 43 & 0.63 & 1 & 0.09 & 14 & 0.23 & 2 & 0.15 & 9 \\
\hline Reliance Growth & -0.02 & 35 & 0.27 & 14 & 0.00 & 35 & 0.15 & 14 & 0.04 & 44 \\
\hline Reliance Regular Savings & 0.06 & 21 & 0.25 & 21 & 0.01 & 32 & 0.12 & 27 & 0.10 & 19 \\
\hline Reliance Tax Saver & 0.04 & 26 & 0.28 & 13 & 0.07 & 19 & 0.26 & 1 & 0.03 & 46 \\
\hline Reliance Vision Fund & 0.00 & 31 & 0.16 & 31 & 0.02 & 29 & 0.18 & 7 & -0.05 & 50 \\
\hline SBI Contra & 0.10 & 16 & 0.12 & 37 & -0.05 & 47 & 0.12 & 26 & 0.11 & 15 \\
\hline SBI Magnum Equity & -0.01 & 34 & 0.11 & 38 & 0.04 & 25 & 0.14 & 15 & 0.10 & 24 \\
\hline SBI Magnum Tax Gain & 0.07 & 19 & 0.14 & 36 & 0.00 & 36 & 0.14 & 16 & 0.09 & 27 \\
\hline Sundaram Equity Mulitplier & -0.13 & 49 & 0.06 & 43 & 0.01 & 31 & 0.10 & 34 & 0.07 & 37 \\
\hline Sundaram Growth Fund & 0.00 & 30 & 0.04 & 44 & 0.03 & 27 & 0.10 & 35 & 0.04 & 43 \\
\hline Sundaram Select Focus & -0.22 & 50 & 0.06 & 42 & -0.03 & 44 & -0.01 & 49 & 0.07 & 34 \\
\hline Sundaram Select Midcap & -0.04 & 41 & 0.47 & 3 & 0.05 & 23 & 0.13 & 22 & 0.10 & 22 \\
\hline Sundaram Smile & 0.18 & 7 & -0.22 & 49 & -0.01 & 39 & 0.06 & 44 & 0.11 & 17 \\
\hline Sundaram Tax Saver & 0.41 & 2 & 0.31 & 11 & -0.09 & 49 & -0.01 & 50 & 0.23 & 1 \\
\hline Tata Equity 0pp & -0.01 & 32 & 0.36 & 7 & -0.04 & 46 & 0.17 & 8 & 0.09 & 25 \\
\hline Tata PE Fund & -0.04 & 40 & 0.40 & 5 & 0.02 & 28 & 0.21 & 3 & -0.02 & 49 \\
\hline Tata Pure Equity & -0.07 & 46 & 0.25 & 22 & -0.02 & 40 & 0.15 & 12 & 0.08 & 28 \\
\hline Tata Tax Saving & 0.01 & 29 & -0.24 & 50 & 0.04 & 26 & 0.04 & 47 & 0.04 & 41 \\
\hline UTI Dividend Yield & 0.12 & 13 & 0.37 & 6 & 0.12 & 4 & 0.10 & 37 & 0.03 & 45 \\
\hline UTI Equity & 0.11 & 15 & 0.25 & 20 & 0.11 & 10 & 0.14 & 19 & 0.10 & 23 \\
\hline UTI Midcap & 0.18 & 6 & 0.14 & 34 & 0.07 & 21 & 0.09 & 40 & 0.16 & 6 \\
\hline
\end{tabular}




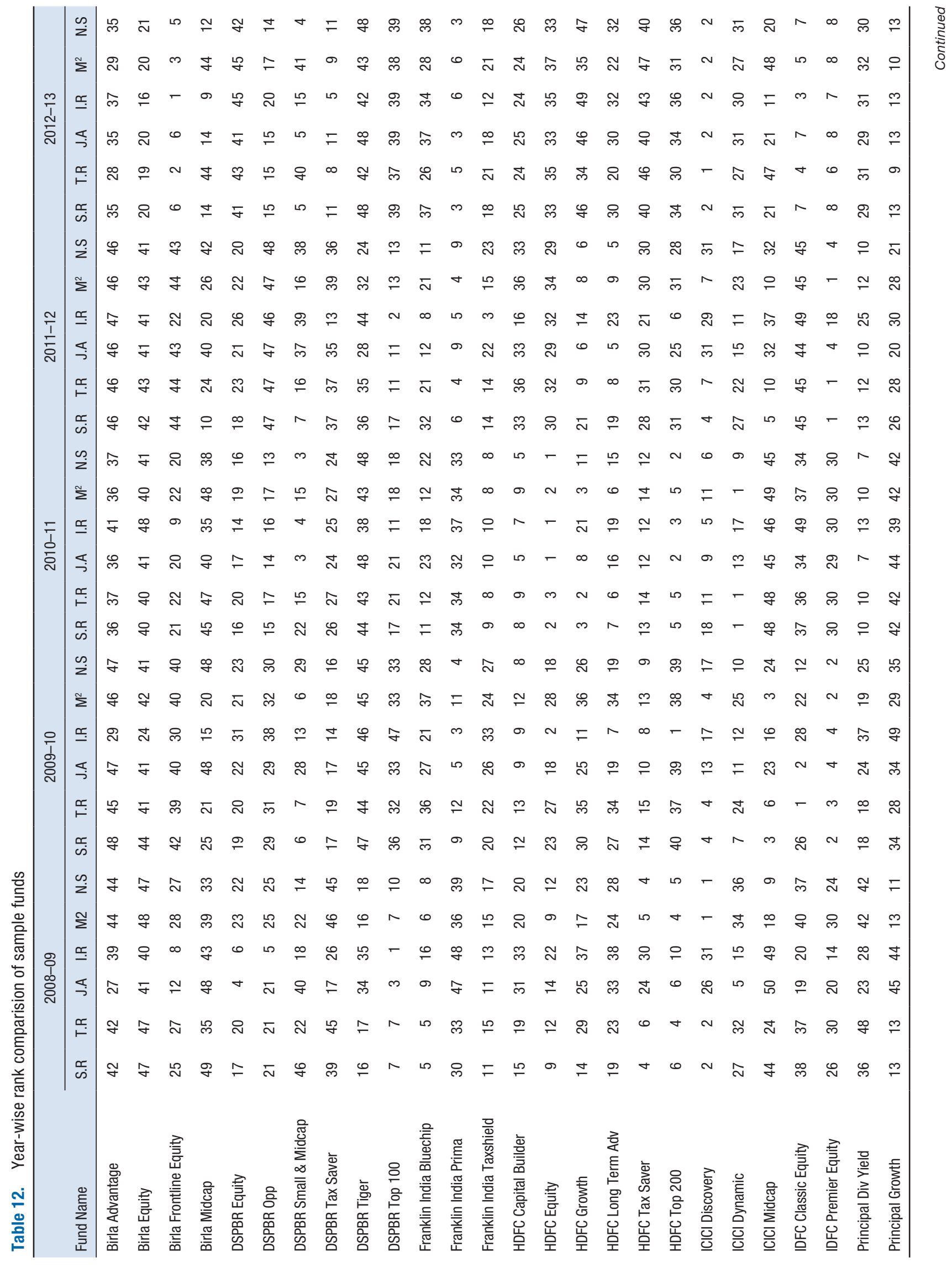




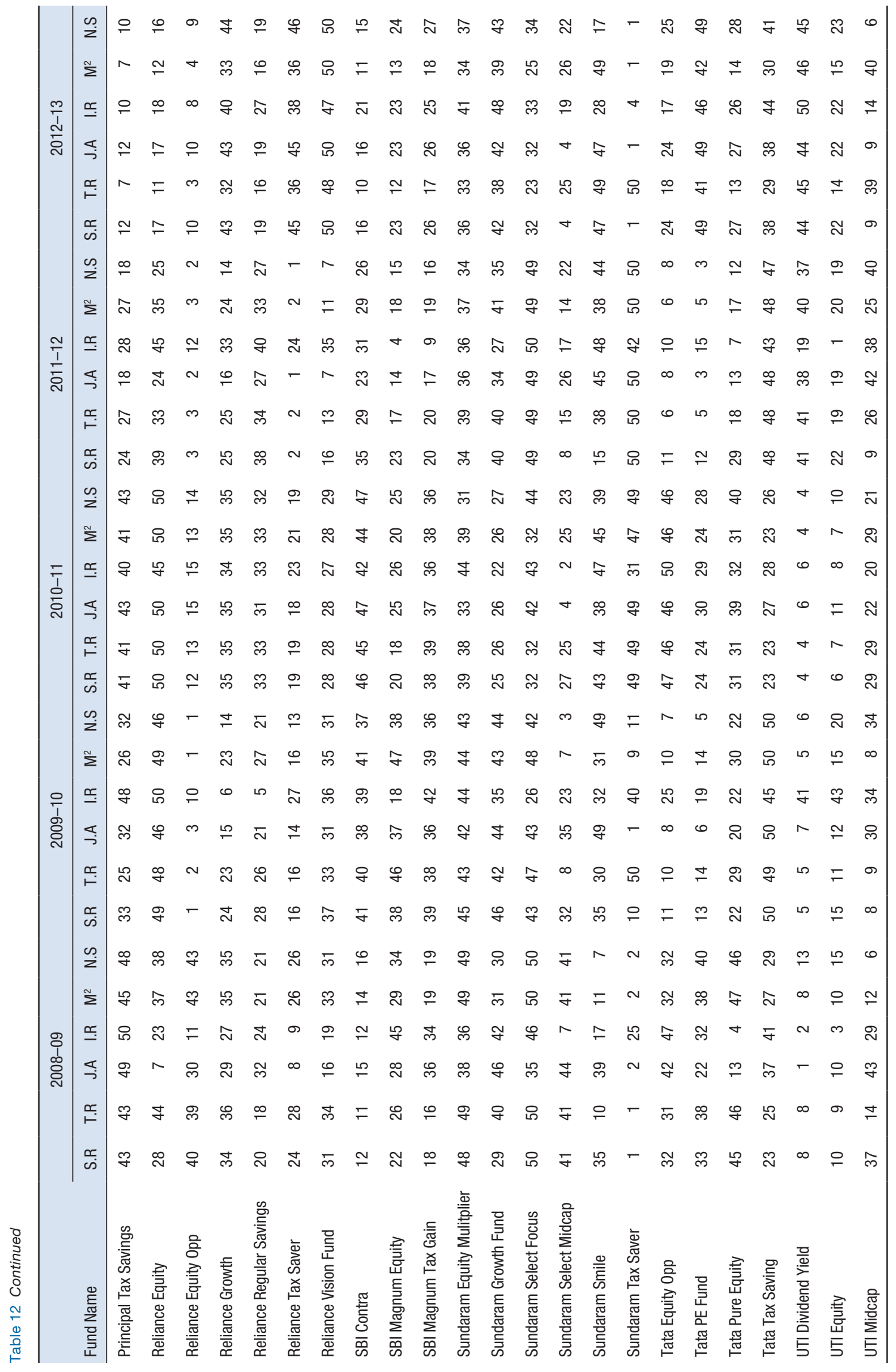




\section{References}

Ang, J. S., Chen, C. R., \& Lin, J. W. (1998). Mutual fund managers' efforts and performance. Journal of Investing, 7, 68-75.

Barron, J. M., \& Ni, J. (2013). Morningstar ratings and mutual fund manager turnover. Journal of Applied Finance, 23, 95-110.

Chang, E. C., \& Lewellen, W. G. (1984). Market timing and mutual fund investment performance. The Journal of Business, 57,57-72.

Chopra, M. P. (2011). Do Indian mutual fund managers select the stock and time the market correctly? IUP Journal of Applied Finance, 17, 77-86.

Cuthbertson, K., Nitzsche, D., \& O’Sullivan, N. (2012). False discovery in uk mutual fund performance. European Financial Management, 18, 444-463.

Davis, J. L. (2001). Mutual Fund performance and manager style. Financial Analysts Journal, 57, 19-27.

Goodwin, T. H. (1998). The information ratio. Financial Analysts Journal, 54, 34-43.

Graham, J. R., \& Harvey, C. R. (1994). Market timing ability and volatility implied in investment advisors' asset allocation recommendations. National Bureau of Economic Research Working Paper 4890.

Grossman, S., \& Stiglitz, J. (1980), On the impossibility of informationally efficient markets. American Economic Review, 70. 393-408.

Huang, J., Sialm, C., \& Zhang, H. (2011). Risk shifting and mutual fund performance, Review of Financial Studies, 24, 2575-2616.

Indro D. C., Jiang, C. X., Hu, M. Y., \& Lee, W. Y. (1998). Mutual fund performance: a question of style, Journal of Investing, 7, 46-53.

Ippoliti, A. R. (1993). On studies of mutual fund performance. Financial Analysts Journal 49, 42.

Kent, D., Grinblatt, M., Titman, S. \& Wermers, R. (1997). Measuring mutual fund performance with characteristic-based benchmarks. The Journal of Finance, 52, 1035-1058.

Kumar, R. (2012). Market timing, selectivity and mutual fund performance: an empirical investigation of selective equity diversified schemes in India. IUP Journal of Financial Economics, 10, 62-84.

Patil, S. R., \& Rao, P. (2011). An empirical study on performance of mutual fund in India. Journal of Contemporary Research in Management 6, 91-103.

Rao U. (2000). Market timing and mutual fund performance. American Business Review, 18, 75-79.
Sharpe, W. F. (1966). Mutual fund performance. Journal of Business 39, 119-138.

Treynor, J. L. (1966). How to rate management investment funds. Harvard Business Review, 43, 63-75.

Treynor, J. L., \& Black, F. (1973). How to use security analysis to improve security selection. Journal of Business, 46, 66-86.

Vos W. (1997). Measuring mutual fund performance. Canadian Investment Review, 10, 33.

\section{About the Author(s)}

Prof. Janaki Ramudu is a doctorate in finance, a post-graduate in Business management, and a graduate in Commerce, from Sri Venkateswara University, Tirupati, India, and has twenty four years of experience in teaching, research, and administration in the field of Management Education. Dr. Ramudu is currently a Professor of Finance at Alliance University School of Business, Bangalore, India, and a member of several professional associations like All India Management Association, Indian Society for Training and Development, and Consultancy Development Cell. He has a fair take of research and publications having published in journals like Journal of Accounting and Finance, South Asian Journal of Management, World Journal of Social Sciences, and Australian Journal of Business Management and Research. He can be contacted at: janakiramudu.p@ alliance.edu.in

Prof. Krishna Kumar is currently an Assistant Professor of Finance at Alliance University School of Business, Bangalore, India. With over twenty years of experience in industry and academics, Prof. Krishna Kumar teaches courses related to finance and commerce. An alumnus of the Institute of Chartered Financial Analysts of India, Hyderabad, India, Prof. Kumar holds a Master's Degree in Financial Analysis and a professional certification in finance. Being an industry professional, Prof. Kumar has a rich and varied experience in financial services and his research interests are financial markets, mutual funds, and corporate finance. He can be contacted at: krishnakumar.k@alliance.edu.in 


\begin{tabular}{|c|c|c|c|}
\hline Index & Funds selected & Index & Funds selected \\
\hline \multirow[t]{12}{*}{ BSE 100} & DSPBR Tiger & CNX 500 & DSPBR Equity \\
\hline & DSPBR Top 100 & & DSPBR 0pp \\
\hline & UTI Dividend Yield & & DSPBR Tax Saver \\
\hline & UTI Equity & & Franklin India Prima \\
\hline & SBI Magnum Tax Gain & & Franklin India Taxshield \\
\hline & SBI Contra & & HDFC Capital Builder \\
\hline & Reliance Equity 0pp & & HDFC Equity \\
\hline & Reliance Growth & & HDFC Tax Saver \\
\hline & Reliance Regular Savings & & Sundaram Equity Mulitplier \\
\hline & Reliance Tax Saver & & Principal Div Yield \\
\hline & Reliance Vision Fund & CNX Midcapcap & Birla Midcap \\
\hline & Principal Large Cap & & DSPBR Small \& Midcap \\
\hline \multirow[t]{10}{*}{ BSE 200} & Birla Advantage & & ICICI Discovery \\
\hline & Birla Equity & & ICICI Midcap \\
\hline & Birla Frontline Equity & & UTI Midcap \\
\hline & HDFC Top 200 & & Sundaram Smile \\
\hline & IDFC Classic Equity & CNX Nifty & ICICI Dynamic \\
\hline & Tata Equity 0pp & & Sundaram Select Focus \\
\hline & Sundaram Growth Fund & & SBI Magnum Equity \\
\hline & Sundaram Tax Saver & & Reliance Equity \\
\hline & Principal Growth & SENSEX & Franklin India Bluechip \\
\hline & Principal Tax Savings & & HDFC Growth \\
\hline BSE 500 & IDFC Premier Equity & & HDFC Long Term Adv \\
\hline \multirow{3}{*}{$\begin{array}{l}\text { BSE } \\
\text { Midcap }\end{array}$} & Sundaram Select Midcap & & Tata PE Fund \\
\hline & & & Tata Pure Equity \\
\hline & & & Tata Tax Saving \\
\hline
\end{tabular}


